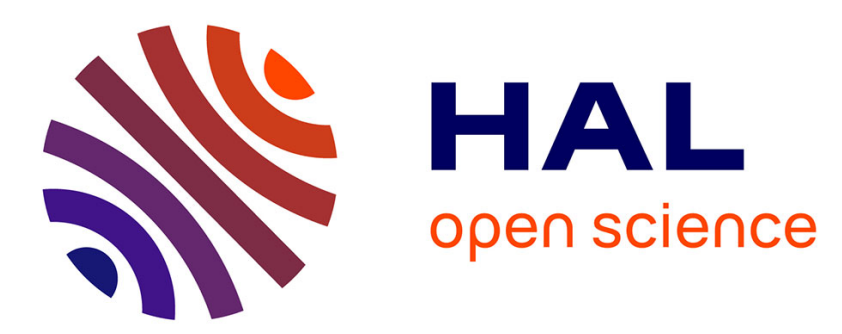

\title{
Petits arrangements avec la diversité. Le recrutement entre marché et mobilisation salariale \\ Sylvie Monchatre
}

\section{To cite this version:}

Sylvie Monchatre. Petits arrangements avec la diversité. Le recrutement entre marché et mobilisation salariale. Revue française de sociologie, 2014, http://www.cairn.info/resume.php?ID_ARTICLE=RFS_551_0041. halshs-01099031

\section{HAL Id: halshs-01099031 https://shs.hal.science/halshs-01099031}

Submitted on 5 Jan 2015

HAL is a multi-disciplinary open access archive for the deposit and dissemination of scientific research documents, whether they are published or not. The documents may come from teaching and research institutions in France or abroad, or from public or private research centers.
L'archive ouverte pluridisciplinaire HAL, est destinée au dépôt et à la diffusion de documents scientifiques de niveau recherche, publiés ou non, émanant des établissements d'enseignement et de recherche français ou étrangers, des laboratoires publics ou privés. 


\section{Petits arrangements avec la diversité. Le recrutement entre marché et mobilisation salariale Sylvie Monchatre ${ }^{1}$ (Université de Strasbourg - Sage - UMR 7363)}

\section{Résumé :}

La diversification des recrutements constitue la réponse managériale apportée au renforcement de la législation anti-discrimination. Elle place les recruteurs devant l'injonction paradoxale de devoir à la fois ignorer et valoriser les " différences". Comment peuvent-ils y parvenir sans faire de discrimination positive ? Pour répondre cette question, l'article montre que le recrutement ne se réduit pas à un échange marchand basé sur des " conventions de compétences » mais s'inscrit également dans des logiques de mobilisation différentielle de groupes sociaux. La diversité conduit à promouvoir la présence, dans l'entreprise, de catégories discriminées, sur la base de "conventions d'usages" différenciées selon la présomption de compétence attachée à la source de leur discrédit. L'exemple de l'hôtellerie-restauration de chaîne nous permet de montrer les conditions de mise en œuvre de ces politiques dans un secteur en tension. Si la " diversité " légitime un principe de différenciation de la condition salariale selon les caractéristiques extraprofessionnelles des individus, elle conduit davantage à renflouer une armée de réserve qu'à infléchir les pratiques en vigueur.

Mots-clés : Recrutement, diversité, discriminations, travail, emploi, gestion des ressources humaines, employés, services.

Nombre de signes : 97004 caractères (notes de bas de page et bibliographie comprises hors titre, résumé et mots clés)

\section{Référence :}

Monchatre S., 2014, Petits arrangements avec la diversité. Le recrutement entre marché et mobilisation salariale, Revue Française de sociologie, Vol. 55 (1), p. 41-70, à paraître

\footnotetext{
${ }^{1}$ Je remercie François Vatin pour sa relecture stimulante de la dernière version de ce texte, dont je demeure la seule responsable.
} 
Le recrutement constitue la scène emblématique du marché du travail. Ce lieu de rencontre entre offre et demande ressemble, toutefois, de moins en moins à cet "Eden des droits naturels de l'homme et du citoyen » où " ce qui y règne seul, c'est Liberté, Egalité, Propriété et Bentham » (Marx, [1867] 1969: 178). Certes, les échangistes y viennent pour passer librement contrat sur un pied d'égalité et poursuivre leurs buts égoïstes : valoriser sa force de travail pour le vendeur et acheter la promesse de pouvoir faire usage d'une marchandise génératrice de profit pour l'acheteur. Mais les principes d'égalité et de liberté censés caractériser cet échange peuvent être malmenés par les faits. Dans un contexte de chômage massif, le sort des candidats à l'emploi paraît suspendu au verdict de recruteurs toujours plus soupçonnés d'arbitraire. Ainsi, les pratiques de sélection sont vivement condamnées dès lors qu'elles reproduisent, avec complaisance, l'asymétrie des rapports de travail $^{2}-$ que l'on croyait confinée au cœur du "laboratoire secret de la production ". Et force est de constater que la liberté des recruteurs est de plus en plus encadrée.

De fait, l'action publique s'est étoffée dans ce domaine, mais essentiellement pour prévenir les discriminations - et non le risque d'exclusion. Cette orientation trouve son origine dans des principes économiques d'ordre libéral, affirmés dès le traité d'Amsterdam de 1997 et la création du marché unique européen (Guiraudon, 2004). Elle a été poursuivie par les politiques d'emploi inspirées de la stratégie de Lisbonne et de la stratégie pour l'emploi de I'OCDE de 2006, qui visent l'accroissement des taux d'emploi dans un contexte de vieillissement de la population. L'enjeu est donc d' "activer les inactifs » (Zajdela, 2009), notamment parmi les femmes et les seniors, et de créer les conditions pour que le marché du travail les accepte. Sexisme, racisme ${ }^{3}$ et " âgisme " (Caradec et alii, 2009) sont désormais en ligne de mire, les recruteurs étant priés de ne pas décourager les candidats à l'emploi.

Les recruteurs sont en réalité soumis à une double contrainte, législative et managériale. Non seulement leur action est encadrée par un dispositif juridique qui facilite la dénonciation des discriminations, nous y reviendrons, mais ils sont appelés à contribuer activement à la "promotion de la diversité " (Doytcheva, 2010). Cette "transfiguration managériale de l'antidiscrimination » est le fruit du travail "d'entrepreneurs de la diversité $^{4}$ " ayant milité, depuis le milieu des années 2000, à transformer une contrainte juridique en atout pour la "performance économique " (Bereni, 2009). Prenant appui sur des dispositifs de soft law - charte, label et ANI diversité ${ }^{5}$ - relativement peu contraignants, elle incite les recruteurs à détecter la compétence dans la " différence ". Ces derniers sont dès lors placés devant une injonction paradoxale. II leur faut sélectionner des candidats sans tenir compte de caractéristiques extra-professionnelles qu'il leur faut pourtant diversifier : ils doivent donc à la fois les ignorer et les valoriser.

\footnotetext{
${ }^{2}$ "La gueule de l'emploi » (Cros, 2011), un documentaire qui suscité un certain émoi parmi le public et parmi ses protagonistes, met magnifiquement en lumière la violence que peut comporter une procédure de recrutement. Il n'a d'ailleurs pas manqué de faire réagir également les chercheurs (voir Rieucau, 2012).

${ }^{3}$ Sur les directives européennes « Race » (2000/43/CE) et « Emploi » (2000/78/CE), voir Guiraudon (2004).

${ }^{4}$ Yazid Sabeg et Claude Bébéar ont notamment promu la notion dans deux rapports fondateurs (Bereni, 2009). Voir également sur ce point Senac (2012).

${ }^{5}$ La charte de la diversité a été lancée par l'Institut Montaigne en 2004. Le label diversité a été conçu, à l'initiative de l'Etat, par l'association nationale des directeurs de ressources humaines (ANDRH) en 2008 et vise l'obtention d'une certification conforme aux exigences de l'Afnor (Association Française de normalisation). L'ANI (accord national interprofessionnel) sur la diversité a été signé en 2006 et étendu par arrêté en 2008. Si charte et label témoignent de l'engagement des DRH, I'ANI témoigne de celui des "partenaires sociaux " (Lanquetin, 2009).
} 
Comment les entreprises s'emparent-elles de cette injonction paradoxale ? Nous proposons de rendre compte de la manière dont s'en accommodent les chaînes d'hôtellerierestauration pour les recrutements des employés de service. Nous nous appuierons sur une enquête menée auprès de trois chaînes (voir encadré méthodologique) soumises à de forts besoins de recrutement, compte-tenu de l'important turnover qui caractérise leurs emplois de service. Nous montrerons que les politiques de diversité constituent un levier de modernisation de la fonction $\mathrm{RH}$ et du recrutement. Pour autant, elles n'affectent pas seulement la construction des "conventions de compétences" (Eymard-Duvernay et Marchal, 1997) qui se négocient sur la scène marchande du recrutement, mais se placent au service de la mobilisation différentielle de groupes sociaux sur la scène productive. Elles contribuent, à ce titre, à la définition de "conventions d'usages " pour des catégories définies en fonction de caractéristiques extra-professionnelles ${ }^{6}$.

La diversité poursuit ainsi le mouvement, mis en évidence par la sociologie de l'emploi (Maruani et Reynaud, 1993, 2004), d'une différenciation des usages des salariés selon leurs propriétés sociales, ceci au nom de fonctionnalités que nous dégagerons en trois temps. Nous présenterons, tout d'abord, les utilités économiques de la diversité telles qu'elles sont conçues et définies par leurs promoteurs, en l'occurrence par les acteurs de la fonction ressources humaines. Nous verrons qu'au-delà de la promotion d'un "principe de précaution " managérial, elles posent les jalons d'une spécification des usages des salariés selon leurs caractéristiques extra-professionnelles. Nous montrerons, dans un deuxième temps, la manière dont la diversité contribue à la normalisation des dispositifs de recrutement dans des chaînes qui, en réalité, cherchent moins à se diversifier qu'à "structurer la diversité ". Nous analyserons, dans un troisième temps, les ajustements pratiqués par les exploitants face aux principes de diversité normalisatrice des chaînes, leurs enjeux étant moins de " diversifier " que de stabiliser un noyau dur de salariés en mesure de leur être redevables pour prévenir les défections. Nous interrogerons, pour finir, les limites de la contribution des politiques de diversité à la lutte contre les discriminations.

\section{Encadré $\mathrm{n}^{\circ} 1$ : les enquêtes réalisées}

Nous nous appuyons ici sur deux enquêtes menées sur la thématique du recrutement. La première, coordonnée par Y. Fondeur et $\mathrm{G}$. de Larquier du CEE, a été menée dans quatre secteurs: la banque, l'informatique, la grande distribution et l'hôtellerie-restauration. Le rapport final de ce travail collectif est disponible à cette adresse :

http://www.cee-recherche.fr/fr/rapports/72-pratiques-recrutement-selectivite-marche-

travail.pdf. Nous nous appuyons ici sur l'investigation réalisée, avec Michèle Forté, dans le secteur hôtelier, qui s'est déroulée en 2010 et 2011, en Alsace et en région parisienne. Cette enquête nous a permis de mener 25 entretiens avec des acteurs institutionnels (représentants d'employeurs et acteurs de la formation initiale et continue), des intermédiaires publics et privés de l'emploi, des employeurs indépendants et des exploitants appartenant à des chaînes d'hôtellerie (HOT), de restauration de type cafétéria (KFT) et de restauration rapide (RAP). Nous avons en outre réalisé deux observations participantes de séances d'évaluation, une concernant le recrutement de managers (RAP), l'autre

\footnotetext{
${ }^{6}$ Les caractéristiques extra-professionnelles désignent l'ensemble des attributs qui servent à désigner un individu comme membre d'une catégorie générale au nom de la nature ou de la culture. II peut s'agir d'attributs qui lui sont irréversiblement attachés (sexe, couleur de peau, âge, apparence, handicap, etc.), ou qui témoignent de son appartenance à une catégorie socio-culturelle (situation familiale, lieu de résidence, engagements culturels, sportifs, politiques, syndicaux, religieux, etc.).
} 
d' « employés polyvalents de restauration " à l'aide de la méthode de recrutement par simulation (MRS dans la suite du texte), toujours pour RAP. Les trois chaînes auxquelles nous consacrons cet article sont engagées dans des politiques de "diversité ". Notre analyse s'appuie, en outre, sur la documentation fournie, les données chiffrées du bilan social que KFT a accepté de nous communiquer, et la littérature managériale sur la diversité, en particulier les travaux de l'IMS-Entreprendre pour la cité, cette association assurant le secrétariat de la charte de la diversité. Nous nous appuyons également sur une seconde enquête, réalisée en 2011 et 2012, avec des étudiants dans le cadre d'un partenariat entre la Faculté de sciences sociales de I'Université de Strasbourg et l'agence de services spécialisés ${ }^{7}$ de Pôle Emploi du Bas-Rhin, sur la perception de la MRS par les employeurs et les candidats.

\section{Encadré $n^{\circ} 2$ : enjeux méthodologiques du questionnement sur les discriminations}

Une précision s'impose sur les modalités de réalisation d'une enquête qui s'intéresse aux discriminations. Si cette thématique a pu être abordée directement avec les intermédiaires de l'emploi et les recrutés, il était plus délicat d'en faire de même avec les recruteurs. Le risque était de placer leurs pratiques en ligne de mire de l'enquête et de susciter des stratégies défensives, allant du déni à la dénonciation, en passant par le conformisme. Fallait-il pour autant enquêter "à couvert " et dissimuler l'objet de l'investigation ? Nous avons ici fait le choix d'une reformulation de la question initiale, qui portait sur les mécanismes de la sélectivité sur le marché du travail et les risques de discrimination. Nous l'avons recentrée sur les politiques d'emploi qui visent la mise au travail de personnes que les entreprises n'ont pas pour habitude de recruter spontanément: seniors, handicapés, demandeurs d'emploi, candidats "issus de la diversité ". L'enjeu était de comprendre les réponses apportées par les entreprises à ces incitations à recruter des profils " atypiques ". Il était ainsi possible d'aborder ouvertement la question des profils désirés ou indésirables, en lien avec la problématique de recrutement de l'employeur - et ceci sans "tromper " l'enquêté. Cette forme de transparence nous semble faire partie des stratégies d'accès au terrain requises dans un contexte de judiciarisation croissante de la relation d'enquête (Laurens et Neyrat, 2010).

\section{Les utilités de la diversité}

La fonction ressources humaines est celle qui, dans l'entreprise, a le plus intérêt à promouvoir la diversité. Elle y trouve l'opportunité d'affermir sa légitimité ${ }^{8}$, toujours précaire, en même temps qu'un double enjeu à relever. II lui faut, en effet, œuvrer à prévenir les discriminations tout en encourageant une diversification des caractéristiques extra-professionnelles des salariés. Elle s'expose alors aux contradictions inhérentes à cette politique. Car la promotion de la diversité demande moins une "indifférence envers les différences" (Senac, 2012) qu'une mise en valeur de ces dernières qui soit en mesure d'échapper à la discrimination positive (Amadieu, 2008).

La rhétorique managériale de la diversité apporte, précisément, un élément de réponse à cette contradiction. L'objectif affiché n'est pas de favoriser des groupes dominés en leur

\footnotetext{
${ }^{7}$ La MRS, jusqu'ici proposée dans les plateformes de vocation (PFV) de Pôle Emploi, relève désormais d'agences de services spécialisés (ASS).

${ }^{8}$ Phénomène constaté également aux Etats-Unis. Voir sur ce point Dobbin (2009).
} 
permettant d'accéder à l'emploi mais de contribuer à l'utilité collective au sein de l'entreprise (Bereni, 2009). Autrement dit, l'engagement des entreprises en faveur de la diversité ne se place nullement au service d'un enjeu social d'égalité, qui bénéficierait à des minorités défavorisées (Beneni et Jaunait, 2009). Il vise au contraire, et ouvertement, l'intérêt économique des firmes: "lutter contre la discrimination en entreprise n'est pas affaire de compassion mais plutôt d'intérêts bien compris ". Réinterprétation " en positif " de la problématique de la non-discrimination (Doytcheva, 2010 : 429), la diversité convertit une question sociale en enjeu économique.

La conversion de la fonction $\mathrm{RH}$ à cette conception utilitariste de la diversité, condition de sa légitimité dans l'entreprise, se traduit par un travail d'identification de ses utilités, entendues ici comme les bénéfices tirés de l'achat d'un bien (une force de travail) aux propriétés particulières (ses caractéristiques extra-professionnelles). Si ces utilités sont généralement exprimées en termes de "gestion des risques" et de "création d'un avantage concurrentiel » (Robert-Demontrond et Joyeau, 2010 ${ }^{10}$ ), elles se déclinent, dans les chaînes étudiées, à trois niveaux: la prévention du risque juridique associé à la discrimination, la rationalisation des recrutements et, enfin, une différenciation des conventions d'usage des populations discriminées.

\section{La prévention du risque juridique}

Le durcissement de la législation anti-discrimination incite les entreprises à s'engager dans la promotion de la diversité (Junter et Senac, 2011). Il constitue pour la fonction RH un véritable levier lui permettant de proposer des mesures de prévention des risques encourus. Cette dimension juridique occupe une place non négligeable dans les raisons mises en avant par les entreprises signataires de charte de la diversité pour justifier leur engagement ${ }^{11}$. Pourtant, les entretiens menés avec les responsables $\mathrm{RH}$ des chaînes étudiées n'en font que très peu état, ceci d'autant moins que l'évocation d'un risque juridique reviendrait pour eux à reconnaître l'existence de pratiques managériales répréhensibles - qu'ils sont censés combattre. Reste que leurs actions de prévention des risques de discrimination ne manquent pas de s'étoffer, et ceci d'autant plus que ces derniers se sont démultipliés depuis l'adoption de la loi du 16 novembre 2001.

Sous la pression du droit communautaire, le cadre juridique français de la lutte contre les discriminations n'a cessé de s'étendre et de se doter d'instruments visant à renforcer son effectivité (Calvès, 2008). Le droit de la non-discrimination couvre désormais l'ensemble des dimensions de la relation de travail ${ }^{12}$. Il englobe la discrimination indirecte ainsi que le

\footnotetext{
${ }^{9}$ Expression tirée du rapport de Claude Bébéar (2004) "L'entreprise aux couleurs de la France », citée par Bereni (2009: 96).

${ }^{10}$ Pour ces auteurs, si les bienfaits de la diversité n'apparaissent pas scientifiquement prouvés et ne font pas l'unanimité dans les entreprises, l'affirmation de la croyance en la performance de la diversité s'inscrit, en revanche, dans une contrainte forte (p. 31).

${ }^{11}$ Les raisons les plus souvent invoquées pour justifier l'engagement en faveur de la diversité sont par ordre d'importance, l'intégration de la diversité dans une démarche de responsabilité sociale des entreprises (75\%), les raisons éthiques (63\%), la réduction du risque juridique (43\%) et l'amélioration de l'image de l'entreprise (43\%). Précisons que moins d'un tiers (942) des entreprises signataires (3194) ont répondu à ce questionnaire, ce qui atteste de la fragilité de leur engagement. Source : Bilan de la charte de la diversité (2011: 8).

12 G. Calvès (2008 : 35) souligne qu' « avec la loi du 16 novembre 2001, il s'est étendu à la totalité de la relation de travail : les salariés sont désormais protégés contre les discriminations qui pourraient apparaître "en
} 
harcèlement moral (depuis 2002) et la liste des critères de discrimination prohibés n'a cessé de s'étendre. Par ailleurs, la charge de la preuve de la discrimination n'incombe plus uniquement à la victime, qui bénéficie en outre, depuis 2001, de possibilités d'aide par voie syndicale, par l'inspection du travail et, depuis 2004, par l'ex-Halde (devenue le Défenseur des Droits en 2011). Ce renforcement de l'équipement juridique des victimes potentielles de discrimination expose les relations de travail à l'éclosion de conflits pouvant entraîner des actions en justice préjudiciables pour l'entreprise.

Un tel contexte encourage les acteurs de la fonction Ressources humaines à promouvoir, en interne, ce qui s'apparente à un principe de précaution dans les relations de travail. Dans les chaînes étudiées, la promotion d'un management préventif emprunte plusieurs voies. Au sein de la chaîne de cafétérias KFT, le directeur du " développement humain " revendique d'en avoir fait un combat personnel avant l'heure: "Peu de temps après que je sois arrivé [dans l'entreprise, fin 1999], j'ai pu, c'était une chance inouïe, licencier un directeur qui avait tenu en réunion de recrutement des propos racistes. Et donc je m'en suis servi pour dire stop. " (Responsable $\mathrm{RH}-\mathrm{KFT}^{13}$ ). Son engagement relève de principes de "fraternité chrétienne " (Doytcheva, $2010: 431$ ) qui le conduisent à se montrer très critique envers la notion même de diversité : "Ce n'est pas un concept qui me va, la diversité. Ce qui me va, c'est motivation et compétences». La lutte contre les discriminations revient ici à promouvoir une culture d'entreprise en termes d' "amour des gens", de "partage ", de " confiance ", de "progrès ". Ces valeurs restent au cœur de la "vision 2020 ", adoptée par KFT en 2011 et assortie d'une véritable éthique du service associé à la restauration populaire: "Donc on recherche des candidats qui aiment les gens, qui vont partager les valeurs de l'enseigne (...). La restauration, c'est faire plaisir, que les personnes viennent passer un bon moment » (Expert recrutement, KFT).

Les responsables RH des autres chaînes situent la lutte contre les discriminations sur un registre plus pragmatique et instrumental. HOT affiche ainsi une approche " business " de la diversité (Doytcheva, 2010 : 432), au nom d'enjeux commerciaux, d'adéquation entre "le personnel de l'entreprise et sa clientèle ", mais également financiers, la RSE (Responsabilité sociale des entreprises) étant de plus en plus prise en compte par les agences de notation. II s'agit ici de prévenir le risque juridique par une modernisation du management qui passe surtout par la formation. HOT appartient au GNC (Groupement national des chaînes) qui a conçu, avec l'aide du $\mathrm{FAFIH}^{14}$ une formation en e-learning sur le double thème de la lutte contre les discriminations et de la diversité pour les managers (voir encadré 3 ). Ces opérations de sensibilisation ne concernent pas seulement le recrutement, mais prennent acte de l'étendue du droit de la non-discrimination en inscrivant la prévention des risques dans l'ensemble des composantes de la gestion des " collaborateurs».

Encadré 3 : Texte de la présentation vidéo d'une formation en e-learning pour managers conçue pour le GNC (Groupement national des chaînes)

"Dans une société où chacun est différent, en comprendre sa diversité et savoir reconnaître les discriminations autour de nous...

matière de rémunération, de formation, de reclassement, d'affectation, de qualification, de classification, de promotion professionnelle, de mutation ou de renouvellement de contrat" ".

${ }^{13}$ Cet ancien DRH a exercé pendant douze ans chez KFT et venait de s'en retirer au moment de notre enquête.

${ }^{14}$ Le Fafih est l'Organisme Paritaire Collecteur Agréé (OPCA) de l'Hôtellerie, de la Restauration et des activités de loisirs. 


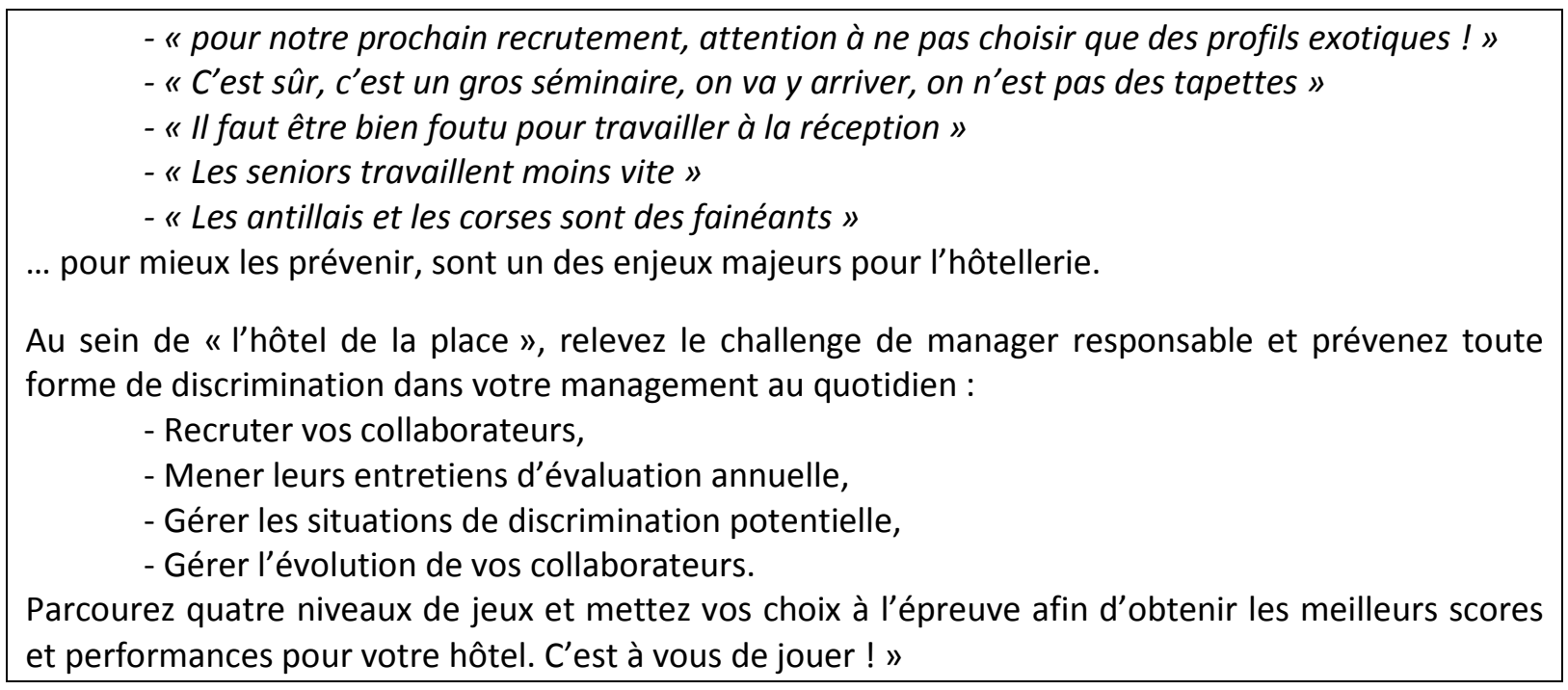

Source : FAFIH

Cet appel à un " management responsable » exprime à mots couverts un regard critique sur les pratiques des responsables opérationnels dans les trois chaînes étudiées. De fait, les professionnels de la fonction RH opposent leur expertise au savoir profane des exploitants. Selon eux, les opérationnels ont " toujours tendance à penser que pour le recrutement, il n'y a pas besoin de formation, c'est une histoire de feeling. (...) Trop souvent, quand ils arrivent en formation, ils disent "celui-là je ne le sens pas, je le sens bien", alors que c'est pas fondé suffisamment sur des critères objectifs " (Expert recrutement, KFT). L'enjeu est donc de leur apprendre à "poser les bonnes questions sans être discriminants": " $\|$ y a des règles à savoir, par exemple, à quelqu'un qui est reconnu handicapé, on ne demande pas de nous dire quel est le type de handicap qu'il a, on lui demande plutôt de nous dire s'il y a des contreindications médicales: "voilà ce que vous allez faire dans le poste, est-ce que vous avez des contre-indications ?" „(Déléguée régionale Emploi, HOT). Ainsi la prévention du risque juridique place les managers en ligne de mire de l'expertise des professionnels de la fonction ressources humaines.

\section{La rationalisation des recrutements}

On sait qu'il existe " deux façons de recruter » (Bessy et Marchal, 2007) : soit par appel au marché du placement, soit par proximité, à l'aide de canaux informels (réseaux, candidatures spontanées, réembauches, ....). Loin de constituer une anomalie, la mobilisation de réseaux est indispensable au fonctionnement du marché du travail. Elle fait intervenir ce " capital social », qui procure aux intéressés de précieuses " ressources d'interconnaissance et d'interreconnaissance" (Bourdieu, 1980), mais également des "liens faibles" (Granovetter, 1973) qui, tout en jouant un rôle de filtre, facilitent l'accès aux opportunités au-delà des cercles familiers. Les réseaux contribuent ainsi à qualifier ou disqualifier les candidatures avant même la rencontre entre offreur et demandeur de travail. S'ils sont avantageux pour les recruteurs, ils relèvent toutefois d'une dynamique homophilique qui les conduit à sélectionner des semblables (Marchal et Rieucau, 2010). La généralité du phénomène peut d'ailleurs entraîner une " discrimination systémique » à l'égard de groupes racisés ou désignés comme culturellement indésirables (Bataille, 1997).

Or, parmi les responsables opérationnels, la tentation de se soustraire au marché est d'autant plus forte que le recrutement n'est pas leur activité principale. De fait, les 
recruteurs ne constituent pas une catégorie générique, unie par des pratiques de jugement les soumettant au même type d'obligations ${ }^{15}$. Nous distinguerons ici les "professionnels", c'est-à-dire les " experts » en recrutement de la fonction $\mathrm{RH}$, des "recruteurs occasionnels " que sont les exploitants (directeurs d'établissements, adjoints de direction, managers). Dans les chaînes étudiées, le rôle des experts est de venir en appui à ces derniers pour recruter les salariés appelés à une carrière au-delà de leur établissement d'accueil, ceci à partir du premier niveau d'encadrement. Mais au nom de la diversité, ils développent des formes d'appui, y compris pour les recrutements d'employés, et tendent à s'affirmer comme des " professionnels du marché » pour l'ensemble des recrutements.

Leur appréciation des pratiques profanes est bien résumée dans les "fiches-recrutement " proposées par l'IMS-Entreprendre pour la Cité : «Les opérationnels ont recours massivement à la cooptation, par souci de faciliter le recrutement ou de le sécuriser (...), au risque de favoriser les mécanismes de reproduction sociale, de féminisation/masculinisation ou d'ethnicisation de certains métiers. Les opérationnels ne sont pas conscients des incidences de la cooptation en termes de discrimination et de non-diversité. " (IMS, 2005 : 7). L'enjeu de diversifier les canaux activés vise non seulement à lutter contre le tropisme des opérationnels à cultiver l'entre soi, mais également à mettre en œuvre une sélection équitable que ne garantit pas la cooptation: "Les candidats cooptés sont "dispensés" de certaines étapes de la procédure de sélection, ou leur sélection ne s'opère pas dans les mêmes conditions d'objectivité que celle des autres candidats " (Idem). La cooptation expose l'entreprise à un risque juridique dès lors qu'elle ne permet pas une égalité de traitement des candidats. Les fiches préconisent par conséquent d'aligner les procédures d'évaluation des cooptés sur celles des autres candidats, mais également de réduire la part de recrutements pratiqués par cooptation.

L'enjeu n'est donc pas de mettre fin aux recrutements de proximité mais de renforcer l'homogénéité des épreuves de sélection pour un même type de poste. Il est également de neutraliser les préjugés qui pourraient empêcher la mise en valeur des compétences des candidats. Le risque est en effet que celles-ci soient invisibilisées par des caractéristiques extra-professionnelles susceptibles de discréditer les candidats, là où l'émergence de " conventions de compétences" suppose la prise en compte d'une multitude d'indices d'ordre professionnel et extra-professionnel. Diversifier les profils demande aux recruteurs de pondérer différemment ces indices, voire de revisiter leurs « conventions de qualité du travail " (Larquier et Salognon, 2005), autrement dit, de revoir leurs exigences et leurs épreuves d'évaluation. C'est précisément ce que proposent les méthodes de recrutement sans CV, qui font partie des instruments-phares de la lutte contre la discrimination.

Toutes les chaînes étudiées ont signé un accord avec Pôle Emploi pour l'utilisation de la méthode de recrutement par simulation (MRS). Labellisée par la Halde en 2007, la MRS tend à constituer une "vitrine des pratiques de recrutement " (Bouchardeau et Capelier, 2010). Elle contribue à la diversité en donnant leur chance à des candidats habituellement exclus des procédures classiques de tri sur diplômes et expérience. Ils sont évalués dans le cadre d'exercices permettant de détecter leurs "habiletés" à tenir le poste à pourvoir et les employeurs s'engagent à les recevoir lors d'entretiens de motivation "sans CV ». La

\footnotetext{
${ }^{15}$ Ce qui est notamment le parti-pris adopté par Marchal (1999) et Hidri (2009).
} 
méthode incite ainsi les recruteurs à surmonter leurs préjugés envers des candidats qu'ils n'auraient pas rencontrés par les canaux classiques, en même temps qu'elle élargit le vivier des "employables" par la détection d'aptitudes dissimulées sous des attributs discréditants. A ce titre, elle contribue à la production de " pré jugements d'employabilité » (Lavitry, 2010) qu'il appartient ensuite à l'employeur de confirmer ou non. Toutefois, la promotion de la diversité ne se limite pas à la promotion de compétences révélées par des évaluations prétendant chasser toute forme de subjectivité. Elle s'accompagne d'un travail de catégorisation de segments de main-d'œuvre, entamé bien en amont des opérations de recrutement.

\section{Les usages différenciés des populations discriminées}

Le marché n'est pas le seul horizon des arbitrages réalisés lors du recrutement. L'autonomie de ce dernier doit d'ailleurs être questionnée car il ne relève pas "d'un champ socialement indépendant des autres » (Dubernet, $1996: 14)$. II revendique, certes, un domaine d'action propre au sein de la "gestion des ressources humaines", qui doit être interrogé par les sciences sociales. Pour autant, il ne se limite pas à la production d'une «convention de compétence" sur la scène marchande. II nous faut ici quitter le cadre d'analyse de l'économie des conventions, qui appréhende le recrutement comme une opération de valorisation des qualités d'un bien mis sur le marché (le candidat) et interroge les conventions qui rendent possible cet échange, mais sans questionner les usages auxquels ce bien est destiné ${ }^{16}$. Or, le recrutement $s^{\prime}$ inscrit dans un processus de mobilisation salariale ${ }^{17}$ qui, au-delà du primat de la compétence, contribue au renouvellement des " conventions d'usage " des salariés sur la scène productive. Nous distinguons ces « conventions d'usage » des conventions de compétence en ce qu'elles désignent, non pas les compétences individuellement négociées pour le poste à pourvoir, mais des usages spécifiques de groupes sociaux, inégalement valorisés sur le marché du travail. Elles relèvent, par conséquent, d'un processus de qualification, là où les conventions de compétence relèvent d'un processus de sélection.

Notre hypothèse est que la mise en œuvre des politiques de diversité repose, en amont du recrutement, sur un processus de qualification de segments de main-d'œuvre discrédités, consistant à leur attribuer des vertus productives sur la base de leurs caractéristiques extraprofessionnelles. Ces politiques prétendent rompre avec les formes d'exploitation de groupes sociaux subordonnés dont témoigne une longue et tenace tradition de division horizontale et verticale du travail selon des critères raciaux (Linhart, 1978 ; Bataille, 1997 ; Jounin, 2004 ; Pitti, 2005) ou sexués (Guilbert, 1966; Kergoat, 1982 ; Maruani et Nicole, 1989). Elles mobilisent une rhétorique qui récuse les bénéfices économiques de cette "gestion segmentée de la main d'œuvre " (Bereni, 2009 : 100), au profit du repérage des compétences individuelles au sein des groupes discriminés. L'enjeu serait d'en extraire les individus compétents pour les intégrer aux équipes de travail, qu'une plus grande mixité doit pouvoir dynamiser (Robert-Demontrond et Joyeau, 2010). La démonstration des bienfaits de I'« entreprise inclusive » (IMS, 2008) et de la " différence performante » (Senac, 2012) passe

\footnotetext{
${ }^{16}$ Sa préoccupation étant d'interroger la contribution du recrutement au risque de chômage et d'exclusion (Eymard-Duvernay, 2012).

${ }^{17}$ La mobilisation désigne « les procès sociaux qui sous-tendent les formes de mobilité et qui non seulement les rendent possibles mais encore les articulent en mouvements d'ensemble » (Gaudemar, 1979 : 20).
} 
alors par l'inversion du stigmate (Goffman, 1977) des candidats habituellement discriminés : par un remarquable retournement de situation, ces derniers apparaissent, dans la littérature grise de la fonction $\mathrm{RH}$, comme de véritables perles rares disposées à une loyauté ${ }^{18}$ sans pareil (IMS, 2010).

En réalité, la diversité vise à tirer parti des attributs discréditants de ces candidats aux vertus révélées. Dans les chaînés étudiées, elle vise l'accès à des viviers spécifiques pour alimenter les emplois en tension. Cette spécialisation des canaux de recrutement (Marchal et Rieucau, 2010) permet d'enrôler des candidats qui deviennent captifs d'emplois "sans qualité » en raison de leur propre dépréciation sur le marché du travail. La promesse d'employabilité qui leur est faite peut alors se substituer aux promesses de carrière jusque-là offertes aux exécutants sur les marchés internes (Monchatre, 2007) et, ainsi, contribuer à une gestion " performante " des ressources humaines ${ }^{19}$. II convient toutefois de distinguer deux types de populations: celles qui subissent des discriminations raciales ou sexuées mais dont la compétence est jugée intacte, et celles soupçonnées d'une moindre " employabilité ", tels les handicapés et les seniors. La première catégorie est associée à des "conventions d'usages flexibles " propres aux segments de main-d'œuvre féminine et juvénile, nous y reviendrons. En revanche, les acteurs de la diversité encouragent les managers à proposer ce que nous appellerons ici des " conventions d'usages restreints » aux candidats de la seconde catégorie.

Chez RAP, des "actions de sensibilisation " visent à stimuler l'imagination managériale envers les personnes handicapées - seule catégorie bénéficiant, pour un quota de $6 \%$ des effectifs, d'un traitement préférentiel autorisé par la loi - et à exploiter les opportunités de l'organisation du travail pour intégrer cette "ressource humaine " atypique. Ainsi, " en interne, on fait intervenir des ergonomes et on travaille avec des ESAT [" établissements de service et d'aide par le travail »] (...). Nous, autour du recrutement, toutes les candidatures (...) négatives papier sont traitées par un ESAT. (...) quand on fait intervenir un traiteur, on fait intervenir un ESAT, voilà, toutes ces sensibilisations-là sont importantes pour nous" (Resp. Recrutement, RAP). Ces opérations ${ }^{20}$ sont pilotées par la Mission Diversité-Handicap, chargée des actions en faveur des publics bénéficiaires de ces conventions d'usages restreints : « handicapés » et « seniors » essentiellement ${ }^{21}$.

On comprend ainsi pourquoi, à l'instar de ce qui a été observé dans d'autres entreprises (Doytcheva, 2010 : 429), s'opère une disjonction entre la prise en charge de la lutte contre les discriminations, confiée aux acteurs des ressources humaines, et celle de la " diversité ", confiée à des " missions » spécialisées : les catégories ciblées par ces actions ne bénéficient pas de la même présomption de compétence. Il en résulte une dilution de la question du racisme dans une " approche globale " (Doytcheva, 2009) qui, selon nous, atteste moins d'un désintérêt que d'un intérêt bien compris. De fait, les conventions d'usage des

\footnotetext{
${ }^{18}$ Certains rapports évaluent ainsi les avantages comparatifs des salariés issus de la diversité ("femmes », " handicapés ", " salariés d'origine étrangère ", " seniors ») en termes de turn-over, absentéisme, etc. Voir IMS (2010).

${ }^{19}$ La contribution de la diversité à l'amélioration de la gestion RH est évoquée en 2011 par $41 \%$ des entreprises signataires de la charte de la diversité, cet enjeu étant celui qui gagne le plus en importance par rapport à 2010 (+10\%). Source : Bilan de la charte de la diversité (2011:8).

${ }^{20}$ RAP soutient également une association produisant des spectacles et des films sur la vie professionnelle de travailleurs handicapés, « Hangagés » (www.hangages.org).

${ }^{21}$ Les femmes peuvent également en faire partie, au nom de la conciliation vie professionnelle-vie familiale, mais elles ne sont a priori pas concernées par les actions diversité à la française (Lanquetin, 2009).
} 
populations racisées ne sont pas appelées à évoluer, au contraire : chez RAP, un consensus silencieux prévaut sur la flexibilité qu'il est possible d'en attendre. En revanche, les populations considérées comme ayant un "handicap " sont celles qui bénéficient de la majorité des actions de communication menées au nom de la diversitée ${ }^{22}$. L'enjeu est ici de promouvoir des conventions d'usages restreints pour des catégories dont l'employabilité et la disponibilité ne sont pas considérées comme allant de soi, ce qui relève moins d'une " affaire de compassion » que d'une normalisation des pratiques de GRH.

\section{Diversifier pour normaliser}

Outre qu'elles différencient les conventions d'usages des salariés selon leurs caractéristiques extra-professionnelles, les politiques de diversité visent à maîtriser la composition des stocks et des flux de salariés. Les chaînes étudiées s'engagent, de fait, dans une "diversité normalisatrice " placée au service d'une politique d'image. Leur enjeu est d'agir sur la composition sociologique de leur personnel: "la diversité, ça existe chez RAP depuis toujours, on a 58 nationalités différentes (...) on a de la diversité de profils et d'origines ethniques différentes. Maintenant, la mouvance actuelle, c'est qu'il faut structurer pour le communiquer vers l'extérieur" (Responsable recrutement, RAP). L'objectif est ici de compléter la panoplie "diversité » en direction des seniors et handicapés, en vue de la labellisation: "On a signé la charte de la diversité l'année dernière, et on travaille à obtenir le label pour la fin 2012 ». Pour KFT, l'enjeu est de renforcer son attractivité dans un contexte d'expansion : "Il faut vraiment qu'on développe notre notoriété, on a une image un peu ringarde, la cafétéria, la restauration, c'est difficile, c'est contraignant, c'est le monde des petits chefs, c'est des horaires pas possibles, voilà, ça fait pas envie " (Expert recrutement, KFT).

Mais la diversité ouvre également l'accès à de nouveaux viviers pour les emplois en tension, particulièrement nombreux dans le secteur. De fait, le modèle d'efficience économique de la restauration rapide (RAP), mais également des cafétérias (KFT), repose sur l'intense turnover des «employés polyvalents de restauration ${ }^{23}$ " autour d'une organisation en flux (Nkouitchou Nkouatchet, 2005). Dans la chaîne hôtelière HOT, ce turnover se concentre sur les "employés polyvalents d'hôtellerie " et d'étages. Il en résulte, pour les directions d'établissements, des volumes de recrutement particulièrement élevés, que la fonction $\mathrm{RH}$ vise à réguler à distance. Pour pouvoir afficher les performances d'une diversité normalisée, elle s'emploie à structurer les recrutements à trois niveaux: le contrôle des flux de candidats, l'action sur les canaux et, enfin, l'instauration d'une division du travail de jugement.

\section{Le contrôle des flux}

\footnotetext{
${ }^{22}$ De fait, les « minorités visibles » et « habitants des quartiers sensibles » sont les deux « publics » les moins fréquemment visés par des actions diversité. Source : Bilan 2011 de la Charte de la diversité, p. 10.

${ }^{23}$ Dans les chaînes de restauration, le turn-over des employés est spectaculaire. Il avoisine les $100 \%$ chez KFT, si l'on intègre l'ensemble des départs et les fins de contrats à durée déterminée. Les responsable $\mathrm{RH}$ annoncent, pour leur part, un turn-over calculé uniquement sur les ruptures de contrats, démissions, licenciements, ce qui le porte à $44 \%$ en 2010 , pour $42 \%$ en 2009 et $59 \%$ en 2008 - et permet de le présenter comme un indicateur de l'efficacité de leur action. Pour RAP, il va de 60 à 300\%, selon les établissements, mais varie considérablement selon que les restaurants sont implantés à Paris ou en province.
} 
KFT et RAP recrutent en moyenne annuelle, pour leur centaine de restaurants intégrés ${ }^{24}$, près de 3000 (KFT) à 5000 (RAP) employés de restauration ou équipiers en CDI à temps partiel, avec des recrutements en CDD du même ordre chez KFT, ce qui correspond, en moyenne par restaurant ${ }^{25}$, à une cinquantaine de recrutements annuels. Or, les marchés locaux du travail constituent des réservoirs de main d'œuvre inégaux et opaques pour des responsables RH opérant à distance. II s'agit alors pour eux de structurer des viviers élargis de candidats dont ils pourront repérer les caractéristiques sociographiques. En amont du recrutement, ils construisent des partenariats avec des prescripteurs en mesure de leur proposer les candidats présentant les profils « diversifiés » qu'ils cherchent à recruter.

Cet engagement se concrétise, dans les trois chaînes, par la signature de la Charte de la diversité, du Plan Espoir Banlieue ${ }^{26}$ (HOT et KFT), d'accords d'entreprises sur le handicap et les seniors (HOT et KFT) ainsi que sur la parité hommes-femmes (RAP). La plus zélée dans ce domaine est HOT: "On est signataires de tout! (rires), ça je ne me trompe pas (...) les chartes de l'apprentissage, la diversité, partenariat avec les écoles de la deuxième chance, partenariat avec le Pôle emploi, sur le volet travailleurs handicapés, on a un accord entreprise avec l'AGEFIPH, voilà... ". L'accès aux publics visés passe, quant à lui, par des partenariats institutionnels permettant de construire trois types de viviers: le vivier " demandeurs d'emploi » avec Pôle Emploi, le vivier " jeunes diplômés " avec l'Education nationale ( $\mathrm{HOT}$ et $\mathrm{KFT}^{27}$ ) et, enfin, ce que les responsables $\mathrm{RH}$ appellent le « vivier diversité ", généralement centré sur les travailleurs handicapés, avec l'AGEFIPH.

La mise en œuvre de ces engagements repose sur une organisation déconcentrée de la fonction RH. Chez KFT, trois fonctionnels du pôle national "Ressources " ont " une double casquette » d'expert et d'appui opérationnel auprès de quatorze "réseaux régionaux » rassemblant chacun huit à quinze restaurants. HOT s'est équipée de structures intermédiaires placées au service de ses différentes enseignes. Ses huit "Délégations Régionales Emploi " ont pour mission de " déployer en région toutes ces chartes desquelles on est signataires", avec notamment pour rôle de "faire prendre conscience aussi aux établissements qu'on est dans ... on fait partie d'un grand groupe et qu'on a des obligations aussi, vis-à-vis de tous ces partenariats qui peuvent être signés, auxquels on ne peut pas déroger " (Déléguée régionale, HOT).

Le rôle des experts $\mathrm{RH}$ est de contribuer à la diversité sans la rechercher ouvertement. L'exemple de HOT éclaire particulièrement les arrangements institutionnels résultant de cette injonction paradoxale : "Euh, ben, enfin, c'est très particulier, cette diversité... et Plan espoir banlieues, la charte diversité, c'est recruter des gens issus des ZUS et des $\mathrm{CUCS}^{28}$, c'est

\footnotetext{
${ }^{24}$ Les restaurants des chaînes KFT et RAP sont soit intégrés, soit franchisés. La rationalisation du recrutement concerne uniquement les restaurants intégrés, soit 170 exploitations pour KFT (sur près de 300) et 90 pour RAP (sur 360) en 2010.

${ }^{25}$ Un restaurant rassemble de 35 à 60 salariés, tous à temps partiel pour les employés.

${ }^{26}$ Lancé en 2008 à l'initiative de Fadela Amara, alors Secrétaire d'État chargée de la politique de la ville, ce plan comportait un volet Emploi pour lequel le secteur privé était sollicité.

${ }^{27}$ La rationalisation industrielle pratiquée dans les chaînes de restauration économique les prive du vivier des formations hôtelières, associées à un idéal d'excellence artisanale en décalage avec leurs pratiques dominées par la $5^{\text {ème }}$ gamme (préparations sous vide confectionnées industriellement dans des cuisines centrales).

${ }^{28}$ ZUS : «Zones urbaines sensibles » et CUCS : "Contrats urbains de cohésion sociale » constituent deux dispositifs des politiques de la ville
} 
presque de la discrimination pour moi que de dire "je vais les identifier", c'est un peu bizarre " (Déléguée régionale, HOT). II est alors tentant de demander à Pôle Emploi d'envoyer des " candidats diversité » ou " Plan Espoir Banlieue " : "ça garantit qu'on puisse en recevoir, parce que moi je ne peux pas demander à un candidat "où est-ce que vous habitez ?" » (Ibid).

Or, Pôle Emploi, qui garantit aux demandeurs d'emploi une égalité de traitement, ne saurait pratiquer de présélection en ces termes. Aucune offre d'emploi ne peut mentionner de telles "préférences", y compris pour les handicapés: "tout ce qu'on peut mettre dans l'offre, c'est qu'à compétences égales, l'employeur donnera la priorité à un travailleur handicapé. Notre service juridique a planché un peu sur le sujet et nous a sorti cette formule qu'on peut intégrer à nos offres d'emploi ». En revanche, Pôle Emploi peut contribuer à diversifier les candidats proposés aux entreprises en clair-obscur: " c'est le discours qu'on tient [aux entreprises]: "vous nous dites que vous recherchez un senior, un travailleur handicapé, déposez nous votre offre et nous, on peut s'engager à vous transmettre 'aussi' des candidatures de seniors, de travailleurs handicapés, de moins de 26 ans... mais voilà, on vous en proposera 'aussi' " » (Responsable, INTERMED-1).

Ce jeu de "miroirs et masques" prend fin à l'heure du bilan des opérations. Pôle Emploi peut, pour le compte des entreprises, caractériser ex post les profils recrutés et faire la part des embauches susceptibles de figurer dans le bilan de leurs actions "diversité »: "régulièrement, madame $X-H O T$ m'envoie un mail en me disant "voilà l'état de nos recrutements sur ce trimestre", et elle me demande si, dans le lot des personnes embauchées, il y a des jeunes de moins de 26 ans issus des quartiers. (...) Et dans le cadre d'un échange de bons procédés, je lui dis "effectivement, telle personne fait partie, telle autre..." ". Reste que dans les entreprises, le bilan des engagements "diversité " reste mollement effectué: "Sur "Plan espoir banlieues", je crois qu'il y a un nombre de recrutements prévus, je ne saurai pas vous dire combien, c'est pas réparti par régions, mais je crois que c'est globalement la région parisienne qui recrute le plus et qui va répondre le plus aux engagements. Pour ce qui est des travailleurs handicapés, on est engagés sur 100 recrutements sur deux ans " (Déléguée régionale, HOT). De son côté, KFT, qui estime avoir recruté, en $2008^{29}, 20 \%$ de jeunes issus des quartiers difficiles dans le cadre du « Plan Espoir Banlieue ", envisageait de mettre en place, au moment de notre enquête, un comité de pilotage avec Pôle Emploi, pour assurer plus systématiquement le suivi de la provenance des candidatures.

Le contrôle des flux de recrutements demeure ainsi un exercice délicat, qui demande aux entreprises et à leurs partenaires de se prémunir contre les apories de la diversité. S'il alourdit les procédures, il crée les conditions d'un "rappel à l'ordre " en interne pour rééquilibrer les profils recrutés et atteindre les " quotas " lorsqu'ils existent. Mais il s'inscrit, plus largement, dans une rationalisation du recrutement qui vise à faciliter l'accès au marché du placement.

La diversification des canaux

\footnotetext{
${ }^{29}$ Notons qu'il s'agit d'un décompte très politique puisque ce plan a été lancé en 2008. Depuis, il n'a plus fait l'objet d'un suivi systématique.
} 
Les expert RH encouragent l'accès aux canaux formels pour les recrutements d'employés. Ils prennent en charge, pour leur part, les campagnes de recrutement de l'encadrement à l'échelle nationale ou régionale et opèrent un tri relativement sélectif. Chez KFT, on estime que sur 100 candidatures, 25 sont retenues pour des entretiens et tests, 2 à 3 candidats sont recrutés à l'arrivée, soit une douzaine de candidats évalués pour un recrutement ${ }^{30}$. Les recrutements d'employés ne présentent pas le même degré de sélectivité. Ils sont réalisés dans le cadre d'opérations "programmées" pour les besoins saisonniers, généralement deux fois l'an. Selon l'étendue des besoins, les exploitants font appel à Pôle Emploi et à des intermédiaires spécialisés (dont le Crous) pour toucher des étudiants, à qui ils proposent des CDI ou des CDD susceptibles d'être convertis en "emplois à l'année ". Mais les recrutements s'effectuent également " au fil de l'eau » et du turnover, souvent dans l'urgence. Les canaux mobilisés sont essentiellement informels et de deux types : réseaux d'interconnaissance ou candidatures spontanées, pour des recrutements en CDI à temps partiel. Le tri réalisé est alors bien moindre que pour l'encadrement: le flux de candidatures traitées conduit les établissements enquêtés à recevoir en entretien de l'ordre de 1 à 5 candidats par poste.

La rationalisation des recrutements d'employés passe dès lors par la mobilisation de professionnels réalisant, pour le compte des opérationnels, des présélections de candidatures. Les délégations régionales de HOT jouent précisément ce rôle en interne : " Je reçois, autant que faire se peut, les candidats qui je pense sont intéressants pour nous, de façon à faire des présélections, alors ça peut être dans le vide ou en prévision de, mais ça peut être aussi parce que j'ai besoin de 4 réceptionnistes. Donc là j'active mes réseaux pour obtenir du CV, présélectionner sur la base du comportement, de façon à mâcher le travail aux exploitants" (Déléguée Emploi, HOT). On notera que les chaînes tendent de plus en plus à proposer des services de présélection pour les recrutements d'employés - y compris pour les franchisés chez RAP - dans le cadre d'une mutualisation de services RH à une échelle territoriale.

Mais les présélections sont également déléguées à Pôle Emploi, dans le cadre du recours à la MRS. Cette prestation gratuite de l'opérateur public permet de suppléer aux insuffisances des canaux habituels pour des recrutements en nombre: saisonnalité, ouvertures ou agrandissements de sites, etc. Les canaux s'ouvrent ainsi à un marché du travail élargi, pour des besoins difficiles à satisfaire ou sortant de l'ordinaire. Le développement de ces présélections atteste de la mise en place progressive d'une division du travail de recrutement des employés, qui conduit à faire intervenir séparément experts et opérationnels, tout en créant une interdépendance entre eux dans la construction du jugement.

\section{La division du travail de jugement}

Dans les chaînes étudiées, une division du travail de jugement est déjà à l'œuvre pour le recrutement de l'encadrement, promis à une intégration durable au sein de leur marché interne. Chez RAP, la fonction RH conçoit, à l'aide de consultants externes, des épreuves de sélection « sur mesure " qui combinent « test de personnalité, test de raisonnement, mise en situation managériale, mise en situation commerciale et exercice de groupe " (Exp.-Rec.RAP). Les candidats ayant réussi les épreuves sont reçus en entretien par leur "futur

\footnotetext{
30 Un processus similaire conduit RAP à l'examen de 70 candidatures pour un poste de manager avec 9 évaluations réalisées. Nous ne disposons pas de ces chiffres pour HOT.
} 
patron ", pour une décision finale qui revient aux opérationnels. On retrouve ici, sous une forme raffermie et instituée en division du travail de jugement, ce constat d'Emmanuelle Marchal (1999 : 49) à propos des différences entre recruteurs spécialistes d'un secteur ou d'une technique d'évaluation. Les experts de l'évaluation examinent la personnalité des candidats, tandis que les exploitants évaluent les compétences, dans le cadre d'une complémentarité revendiquée. Or, cette division du travail de jugement, au-delà du " grand partage " des rôles qu'elle instaure, renvoie également à la relation qui unit experts et opérationnels. L'évaluation des experts offre un contrepoids aux évaluations spontanées de ces derniers, soupçonnés de privilégier des semblables qu'ils jugeraient plus loyaux (Hidri, 2009) et " à leur main ". Or, "ce qu'on cherche, c'est d'abord que la personne rentre chez $R A P$, enfin, soit quelqu'un de bon pour RAP, ensuite, bon pour son directeur " (Dir-Rec-RAP). Leur monopole de jugement est ainsi mis à mal, y compris pour les recrutements d'employés.

On sait que dans les chaînes d'hôtellerie-restauration, les pratiques informelles qui prévalent pour les recrutements d'employés bénéficient d'une grande tolérance, en raison de la dépendance des établissements envers leurs marchés locaux du travail, notamment pour la gestion de leurs fluctuations d'activité (Lockyer, Scholarios, 2004). Les recrutements d'employés sont dès lors essentiellement réalisés à partir d'un " jugement simplifié " ou " standard " de tri sur CV et entretien (Larquier et Marchal, 2008). Les politiques de diversité tendent cependant à encadrer ces pratiques à distance, mais selon une autre logique que pour l'encadrement. Le recours à la MRS, qui donne accès au vivier "demandeurs d'emploi ", constitue une bonne illustration de la division du travail de jugement qui s'instaure. Les experts de Pôle Emploi évaluent les " habiletés " des candidats sur la base d'exercices conçus à partir d'une analyse in situ des postes de travail ${ }^{31}$. Leur présélection évalue les dispositions opératoires des candidats à l'aide d'un "jugement contextuel » (Bureau et Marchal, 2009) qui n'est pas laissé à l'appréciation de la hiérarchie preneuse. Ce faisant, la méthode atteste de l'adéquation des habiletés des candidats avec celles requises dans un poste donné, s'inscrivant ainsi dans une logique d'emploi - et non de carrière sur un marché interne. De fait, elle correspond pleinement à la fonction des intermédiaires publics de l'emploi sur le marché du travail, qui est d'intervenir sur le segment des emplois sans perspective (Lagarenne et Marchal, 1995 ; Addison et Portugal, 2002) en même temps que de favoriser le retour à l'emploi des populations stigmatisées pour en être restées durablement éloignées (Aberg, 2001).

Par ailleurs, la MRS contribue à spécifier les usages flexibles attendus des candidats. L'exercice d'évaluation des habiletés des candidats au poste d'employé polyvalent de restauration (EPR) auquel nous avons participé rend bien compte de la dimension prescriptive de ces épreuves. Celles-ci tendent à reproduire la temporalité des situations de travail (ici marquée par l'urgence) mais également à évaluer les dispositions des candidats à exécuter les différentes tâches qui leur sont confiées sans temps morts et au plus près des consignes, tout en leur indiquant les priorités à suivre pour un usage optimal de leur temps de travail (voir encadré 4). La méthode permet ainsi de faire valoir les exigences de l'enseigne et de tester les dispositions des candidats à s'y soumettre (Theurier, 1983). Elle ajoute le recrutement aux sources de prescription qui sont en voie de démultiplication

\footnotetext{
${ }^{31}$ Les exercices sont étalonnés avec des salariés en place puis pondérés pour être soumis à des débutants. Dans les chaines, les exercices conçus pour un emploi sont démultipliés et adaptés dans les établissements.
} 
(Daniellou, 2002), tout en faisant la promotion d'une convention d'usage flexible selon une logique d'emploi - et non de carrière. Elle pose, à ce titre, les jalons d'une différenciation des usages des candidats selon leur canal de provenance.

Encadré 4 : Présélection par MRS de candidats aux postes d'employé polyvalent de restauration La séance d'exercices observés a duré $2 \mathrm{~h} 30$. Elle s'est déroulée dans les locaux de Pôle Emploi et s'est divisée en trois séquences distinctes.

La première séquence correspondait à une mise en situation pratique caractérisée par l'urgence du "rush » et comportant trois exercices : confection de hamburgers (exercice "Exa-plat "), réalisation d'une " recette " de crêpes ou de cookies et enfin, contrôle des dates limites de consommation (DLC) en chambre froide (exercice "fil rouge »). "Donc l'exercice sera rythmé par un CD qui reproduit l'ambiance de votre restaurant, ça démarre avec une tranche calme et une accélération en cours de travail. Le coup de feu de midi. Donc c'est le CD qui vous indiquera ce que vous devrez faire. Soit préparer les assiettes, donc exercice " exa-plat ", quand vous entendrez le bruit d'une imprimante de restaurant, et vous compléterez donc la page exa-plat. Soit vous entendrez votre responsable qui vous dira "prépare-moi des crêpes ou des cookies", et ça, ça sera l'exercice "recettes". Et dès que vous aurez du temps, il faudra checker votre chambre froide ".

L'exercice "Exa-plat " consiste à assembler les éléments rentrant dans la composition d'un burger, ce qui se traduit par la consigne de placer, sur une feuille remplie de figures géométriques, deux points verts à l'intérieur des rectangles, deux points rouge dans les rectangles situés à l'intérieur d'un cadre, un point rouge dans les étoiles, un point vert dans les étoiles situées à l'intérieur d'un cadre et enfin, un point bleu dans toutes les autres figures. Or, le remplissage des figures est tributaire des contraintes de l'ordre de la production: "Le travail doit impérativement s'opérer colonne après colonne. Donc vous voyez, je ne vais pas commencer à faire toutes les étoiles et puis après, tous les rectangles et puis tout le reste. Non, je veux que vous fassiez figure après figure et puis colonne après colonne. Ça c'est une figure à respecter. Sinon, si vous ne faites pas ça, ce sera faux. J'arrive et je vous mets un trait sur la feuille. Parce que vous imaginez, vous êtes en train de préparer vos hamburgers, un client qui commande un hamburger au fromage et l'autre au poisson, et bien on ne va pas faire d'abord 50 au fromage et 50 aux poissons, on prend par rapport aux commandes ". De la même façon, l'exécution doit être sans défaut : "Et pareil, quand vous achetez un hamburger au RAP de Molsheim ou au RAP de Marseille, on a la même dose de ketchup, de fromage, tout est très normé. Donc par cet exercice, on va vérifier que vous êtes en capacité de rester efficaces dans la durée. Donc je veux que les points soient bien au centre des figures, il ne faut pas que ça touche les bords, il faut respecter les couleurs et il faut respecter l'ordre d'arrivée des aliments. D'accord ? "

La deuxième séquence est consacrée à une situation de relation clientèle. L'exercice consiste à recevoir 21 clients (via un $\mathrm{CD}$ ), à choisir, parmi quatre modalités de réponses, la pire et la meilleure, à encaisser le client et lui rendre la monnaie, sachant qu'en cours d'exercice, les candidats sont interpellés par les animateurs qui jouent le rôle d'un client venant les perturber (" Excusez-moi, c'est possible de choisir sa sauce de salade? »). Enfin, l'exercice se termine par le décompte de la caisse. Comme le montrent les consignes retranscrites ici, l'exercice reflète la politique commerciale de la marque, qui consiste, à l'instar de ce qu'a observé F. Hanique (2004) pour les guichetiers de la Poste, non pas à répondre à la demande du client, mais à lui vendre les "formules » en promotion : "Alors ce qu'on attend de vous, c'est établir un contact positif, c'est-à-dire être disponible, réactif, poli, agréable, avec les clients, même en situation de forte affluence, entre $11 \mathrm{~h} 30$ et $14 \mathrm{~h}$, comme tout à l'heure. Vous allez avoir une petite accélération, pour voir vos capacités d'accueillir les clients. Les aider dans leur choix en faisant la promotion des formules du jour. Alors les formules du jour, c'est ça. Aujourd'hui, ce qu'on vous demande c'est de vendre au maximum ces formules du jour. Alors c'est clair, si vous entendez un client qui vous dit "bonjour, je voudrais un jus d'orange, un café et un croissant ", vous n'allez pas lui vendre la formule Balade, qui est un sandwich. Par contre, si c'est proche de ce qu'on vous demande, si vous avez une formule à proposer, c'est ce qu'on vous demande de faire. Promouvoir, c'est-à-dire vendre, au maximum, ça. » 
La troisième séquence était consacrée à un exercice de groupe, visant à organiser une salle en vue de recevoir des clients ayant effectué une réservation. L'enjeu était ici d'évaluer les capacités des candidats à travailler en équipe, car « dans la restauration, c'est très important aussi d'être solidaire entre collègues, donc là on va voir votre comportement ".

Ces exercices rendent parfaitement compte des attentes de polyvalence entre les postes de production (exa-plat, recette et chambre froide) et les postes de relation client (prise de commande et encaissement) qui sont demandées aux employés. Ils indiquent également aux candidats ce que doivent être les priorités en situation de travail, en l'occurrence, savoir s'extraire de la réalisation d'une tâche pour répondre aux sollicitations de la hiérarchie ("Fais-moi des crêpes... ") ou des clients, autrement dit, savoir faire face aux aléas et gérer les situations de dispersion (Datchary, 2010). Mais ils témoignent également de l'exigence d'optimisation de leur temps de travail demandée aux employés : il ne saurait y avoir de temps mort durant le service. Dès qu'un moment vacant se présente, il doit être investi par une tâche : ici, le contrôle de la chambre froide mais, en situation réelle, le ramassage des plateaux et le nettoyage de la salle, en l'occurrence, le "sale boulot $"$.

\section{Une diversité inégalement opportune}

Le développement de ces présélections fait partie des "investissements de formes " qui permettent la présence, sur le marché du travail, de candidats qui en seraient, sinon, écartés. De fait, la discrimination commence par la mise à l'écart du marché du travail (Bataille, 1997), lorsque les recruteurs, dans une logique d'économie du jugement, déduisent les compétences des candidats de caractéristiques extra-professionnelles irréversiblement attachées à leur personne (Eymard-Duvernay et Marchal, 1997). Or, selon les intermédiaires que nous avons rencontrés, les chaînes d'hôtellerie-restauration se montreraient moins discriminatoires que les petits indépendants: "On n'a jamais eu quelqu'un en chaîne qui a dit "je ne veux pas de Noirs", jamais! Autant, la restauration tradi, on nous l'a dit: "je veux pas d'Arabes, hein !" "(Responsable MRS). De fait, leur faible attractivité sur les segments les moins prestigieux du secteur les amène à s'ouvrir aux candidats présentant des profils plus diversifiés.

Pour autant, les chaînes ne sont pas exemptes de pratiques discriminatoires, liées à une différenciation des usages des salariés selon leur caractéristiques physiques. Dans des groupes hôteliers, qui communiquent savamment sur la diversité de leur personnel (Point, 2010), les affectations dans les emplois se différencient selon l'âge, le sexe, mais également la couleur de peau. Des enclaves ethnicisées se donnent ainsi à voir dans les étages (Puech, 2006) et dans les cuisines ${ }^{32}$ : " en cuisine, les mecs, c'est tous des Blacks et les chefs c'est tous des Français. (...) Et au [H*** de HOT], il n'y a pas un seul serveur black, mais par contre tous les plongeurs, c'est des Blacks" (Ex-serveuse, HOT), et plus globalement dans les emplois de back-office, loin du regard du client. De plus, les exigences de la marque passent par une politique vestimentaire qui fait s'immiscer des critères physiques dans les recrutements, par le biais de la taille des uniformes proposés : " dans le dernier [restaurant] où j'étais, c'était 36-38 les pantalons qu'ils avaient achetés et il y avait une fille qui était venue à l'essai, qui était un peu plus costaud, qui ne pouvait pas enfiler son pantalon. Elle n'est pas restée longtemps" (Ex-serveuse, HOT). Le poids des apparences dans les

\footnotetext{
${ }^{32}$ C'est notamment le cas des chaînes de restauration thématique avec service à table, qui peuvent afficher en salle un exotisme " style New-York ", à l'aide d'un savant cocktail d'origines géographiques parmi les serveuses.
} 
recrutements (Hidri, 2008) n'a ainsi nul besoin d'être formulé, il ne résiste pas à l'épreuve de l'activité.

Chez KFT et RAP, la frontière front-office / back-office est moins nette que dans les chaînes de restauration avec service à table. Chez RAP, la polyvalence est de mise et dans les affectations entre "caisse " ou " production ", " on essaie toujours ... pour équilibrer, et puis pareil, ne pas mettre que des femmes en caisse et les messieurs au grill, pareil, on essaie de varier... " (Dir-A-RAP). Reste que chez KFT, certains responsables ne cachent pas que "la diversité, c'est intéressant d'un point de vue commercial ", notamment pour les postes de " take-away" (vente à emporter) où " on essaye d'embaucher des mignonnes! " (Dir-KFT). Ces propos attestent des usages qui sont faits des employés de service en fonction leurs caractéristiques physiques. Le travail "esthétique " qui leur est demandé fait partie du " travail émotionnel » requis dans les services (Hochschild, 1983); il ne saurait toutefois s'y réduire tant il relève d'une demande d'incarnation physique de la marque qui gagne à être étudiée en tant que telle (Warhurst and Nickson, 2007). Les exploitants peuvent ainsi, au nom de leurs contraintes productives, définir leurs besoins en des termes correspondant à des critères prohibés, ce qu'ils sont amenés à contrôler face aux attendus de la " diversité normalisatrice ", comme nous le verrons avec deux cas contrastés.

\section{En quête de loyauté}

Selon l'enquête OFER, le secteur hôtelier réalise $36 \%$ de ses recrutements sur la base d'une seule candidature reçue (Fondeur et Larquier, 2012). De fait, sa faible attractivité le rend tributaire des dynamiques des marchés du travail : "Quand il y a une reprise du marché du travail, forcément pour nous c'est plutôt défavorable, parce que les personnes ne vont pas forcément vers la restauration... " (Exp. Recrutement-KFT). Les exploitants s'estiment à la merci d'un marché du travail local dont ils dépendent entièrement. Tributaires d'une "ressource humaine" dont le turnover structurel fait d'eux des Sisyphe guettés par l'absurde, ils vivent ce renouvellement récurrent comme une menace pour l'équilibre, fragile, d'équipes de travail exposées à un fort absentéisme. Le risque est, pour eux, de recruter dans l'urgence et de "faire des bêtises ", voire de se tromper : "ce qui est pire! Je dis toujours : on ne recrute pas quand on a faim ! " (Dir-B-RAP).

Par ailleurs, les exploitants définissent essentiellement leurs besoins en termes de "disponibilité temporelle" (Alaluf, 2000; Devetter, 2006): "On a un échéancier d'embauche, c'est un document Excel qui nous donne les volumes d'heures à pourvoir. Par exemple, pour faire un chiffre d'affaire de 7000 euros, il me faut 100h de travail à repartir... donc moi je raisonne en heures. Si avec l'équipe en place je ne peux en assurer que 80 , il m'en manque 20: il faut donc un équipier pour 20h" (Dir-B-RAP). Or, les responsables RH récusent cette approche des exploitants: "On ne recrute plus des créneaux horaires, on recrute des compétences! » (Dir-Rec-RAP). C'est ainsi que chez KFT, les exploitants ont été équipés d'un système de "gestion prévisionnelle de l'absentéisme " mais aussi d'une "carte d'expertise " qui leur permet de planifier leurs besoins de recrutement en quantité et en qualité.

Or, le fort pouvoir de défection des salariés et la crainte de ne pas trouver de candidats disponibles pour leurs horaires décalés conduisent les recruteurs à reformuler leurs exigences. Bien qu'ils estiment ne pas toujours être en mesure de sélectionner, ils opèrent 
néanmoins un tri dont la compétence est souvent exclue : "Parce que pour travailler chez $R A P$, il ne faut pas grand-chose. On forme les gens, c'est pas un métier... je pense qu'une grande majorité peut travailler chez nous " (Dir-A-RAP). Leurs exigences s'expriment alors en termes de motivation. Ce " mot-valise » renvoie à l'adhésion sans réserve des candidats aux objectifs de l'entreprise (Cartier, 2001 : 80), ou encore à leur disponibilité temporelle, qui doit être sans faille (Papinot, 2011). Mais ici, la motivation présente une dimension supplémentaire: "des gens motivés, [il faut] qu'on ressente que... c'est pas le tout de se dire motivé, il faut qu'on voie qu'ils ont envie de venir. Il faut qu'il y ait l'envie, ce qu'on cherche ça va être des gens fiables, mais trouver quelqu'un de fiable par un entretien, c'est ... c'est pas évident... " (Dir-A-RAP). Autrement dit, les recruteurs recherchent des " gens " loyaux, sur qui ils veulent pouvoir compter.

Or le marché n'est pas le meilleur candidat pour satisfaire cette demande. II amène des candidatures inconnues jugées difficiles à évaluer lors d'un simple entretien. Le recours aux réseaux de proximité est donc bien plus prisé. Les candidatures spontanées, suscitées par le bouche-à-oreille ou provenant de recommandations de salariés en place, sont particulièrement encouragées: "Ils vont nous amener quelqu'un de fiable parce qu'ils savent que ça va être leur collègue demain, donc ils ne vont pas ramener quelqu'un qui va être absent et qui va ... donc oui. On va favoriser les amis, les connaissances, le cousin... " (Dir-A-RAP). Les recommandations contribuent à inscrire le recrutement dans un échange social traversé de rapports de don et de dettes (Godbout, 1995 ; Richebé, 2002), susceptible de contribuer à prévenir le risque de défection (Petersen et alii, 2000). Leurs vertus sont telles qu'elles peuvent être érigées en "système de parrainage " formalisé et monnayé, comme chez RAP où il a fonctionné pendant un temps : "il y avait des primes qui étaient versées pour des gens qui arrivaient à faire rentrer des connaissances » (Dir-A-RAP).

Pour autant, réseaux et recommandations ont leurs limites. Les exploitants veillent à ce que les candidatures émanent de salariés de confiance. Ils redoutent les liens trop forts entre collègues, susceptibles de perturber le service et d'aller à l'encontre d'un respect orthodoxe des consignes. De même, ils veillent à empêcher une trop grande proximité avec la clientèle, propice aux vols ou aux dons de marchandise lors des passages en caisse pour "les copains et les copines du coin qu'on ne va pas faire payer". Aux alentours de banlieues réputées difficiles, les responsables refusent d'embaucher les candidats domiciliés à proximité " pour éviter de créer des ghettos et d'importer les problèmes de la cité dans le restaurant " (Dir-BRAP). Les réseaux amènent donc des candidats sélectionnés moins pour leurs compétences que pour leur capacité à ne pas nuire à la bonne marche du service.

Ce principe de sélection par la négative conduit à éliminer a priori les profils indésirables et ouvre la voie aux discriminations. II s'inscrit plus largement dans un travail de catégorisation " indigène " des candidats sur la base de leurs caractéristiques extra-professionnelles. A l'instar de ce qu'ont observé Balazs et Faguer (1979: 51) chez les petits artisans recherchant des jeunes pour leurs emplois non qualifiés, les exploitants portent ici un "surcroît d'attention " aux " caractéristiques sociales » des candidats. Ils recherchent des jeunes et ne recrutent guère au-delà de 40 ans, au nom du caractère éprouvant des postes à pourvoir : "le travail est difficile, parce que c'est quand même physique, c'est pas un poste d'employé de bureau " (Adj.-Dir-KFT). Leur préférence va ainsi aux "cadets sociaux ", appartenant à des groupes subordonnés et se montrant $a$ priori disposés à se soumettre à des exigences 
multiformes: polyvalence, remplacement de collègues absents ou réalisation du "sale boulot ", à savoir "nettoyer et nettoyer pas forcément des surfaces de tables, mais ça va aller du joint de carrelage au plafond... " (Dir-B-RAP). Enfin, l'apparence joue un grand rôle (Hidri, 2009). Les candidats doivent être "propres", "pas débraillés" (HOT) et les exploitants sont prompts à lire dans leur " hexis corporelle " les indices d'une personnalité adaptée aux exigences de l'emploi (Cartier, 2001) : "il nous faut des gens souriants et qui sont à l'aise, on ne peut pas prendre des gens timides, c'est pas possible » (Dir-A-RAP).

La mobilisation de salariés selon ces conventions d'usages flexibles s'inscrit dans des stratégies de neutralisation du turn-over qui se montrent inégalement adaptées à une diversification "normalisée " des recrutements. Deux cas de restaurants de province nous permettront de l'illustrer: l'un pratique un "recrutement endogénéisé ${ }^{33}$ ", selon une logique de stabilisation de candidats de l'uniformité allant à l'encontre de toute diversification; l'autre recrute sur un mode plus exogène et privilégie une stabilité polyvalente davantage propice à la diversité requise.

\section{Recrutement endogène, fermeture du marché et entraves à la diversité}

Ce restaurant de KFT présente un mode de recrutement endogène en ce que les candidats sont recherchés avant tout en interne : "ce qui marche le mieux, c'est le bouche-à-oreille Des gens recommandés par le personnel ? Oui, tout à fait, oui. C'est souvent, soit des membres de la famille, des cousins, des cousines, des neveux, des nièces, donc ou tout simplement des connaissances " (Adj.-Dir-KFT). Cette politique d'embauche des enfants du personnel perdure dans le restaurant indépendamment du changement de directeur intervenu récemment, et grâce à $M m e ~ Y$, chargée des ressources humaines et plus particulièrement des recrutements. Avec ses trente ans d'ancienneté, cette adjointe du directeur, issue de la promotion interne après avoir commencé sur le tas sans diplôme, pourrait faire partie de ces "reines » décrites par $M$. Waelli parmi les caissières de supermarché, qui doivent à l'entreprise une promotion sociale inespérée.

Sa longue expérience a permis à Mme $\mathrm{Y}$ de mettre en place ce qu'elle présente comme un échange de bons procédés. Elle distingue deux types d'usage des employés selon leurs propriétés sociales. Les mères de famille d'âge intermédiaire sont préférentiellement affectées à la caisse ou aux autres postes sur lesquels " on doit mettre des gens de confiance ". Quant aux "étudiants", même si dans cette enseigne, "on n'est pas autant axés sur [eux] que la concurrence", ils sont très prisés car "on augmente leur nombre $d$ 'heures pendant les vacances scolaires, ça les arrange et nous aussi, et en plus, ils se forment tous seuls!". Ces caractéristiques extra-professionnelles des salariés permettent également une gestion différenciée des plannings. A côté des étudiants qui « demandent à ne travailler que le soir », des aménagements sont prévus pour les mères de famille : "Chez

\footnotetext{
${ }^{33}$ Nous empruntons cette formule à Gaudemar (1979), qui distingue les différentes phases du capitalisme ayant permis de passer du recrutement d'une main d'œuvre exogène, socialisée en dehors des institutions de la société salariale, à celui d'une main d'œuvre endogène qui au contraire, en est issue. L'endogénéité des recrutements prend des formes différentes selon le type de structure. On l'observe dans les pratiques des indépendants de la restauration, qui privilégient les candidats formés par les institutions de la profession ou issus de réseaux de proximité. Dans les chaînes, elle se manifeste par des pratiques de cooptation qui privilégient les candidats appartenant aux mêmes cercles de sociabilité que les salariés. Voir également sur ce point Forté et Monchatre (2013).
} 
KFT, les mamans sont prioritaires pour le mercredi... ". Le binôme " mamans-étudiants » est présenté comme une formule gagnante.

Le recours au marché du placement concerne uniquement les offres de CDD pour étudiants. Pour eux, Mme Y dispose, au sein du "Relais Emploi " local qui travaille avec le Crous, d'une interlocutrice qui a "bien compris [ses] besoins » et lui présente des étudiants lors de ses deux opérations annuelles de recrutement de saisonniers pour les vacances. Ce recours au marché ne concerne donc que les emplois situés à la périphérie du noyau dur de salariés "permanents", recrutés, pour leur part, par candidatures spontanées. Celles-ci sont acheminées par le bouche-à-oreille, mais peuvent également permettre de recevoir des "gens complètement nouveaux ". De fait, Mme $\mathrm{Y}$ a décidé de s'ouvrir à des candidats inconnus, mais limite le risque encouru en ne retenant de sa pile de candidatures spontanées que des candidats présentant une expérience dans la restauration : "ils savent comme ça exactement où ils mettent les pieds!". L'expérience demeure ici le "critère royal » qui permet une sélection sur la base du « déjà fait » (Dubernet, 1996).

Le caractère endogène des recrutements se manifeste ainsi par la quête de candidats recommandés par des salariés de l'organisation ou par des pairs de la profession. Elle conduit à terme à la stabilisation d'un noyau dur qui permet à ce restaurant d'afficher un des taux d'ancienneté les plus élevés du groupe. Le compromis salarial tacite qui en résulte est la récompense de la loyauté sur le long terme, les salariés les plus anciens se voyant offrir des contrats de travail à temps plein mais également des postes aménagés : "... nous, on privilégie les gens qui sont déjà dans l'entreprise. Parce qu'on a pas mal de gens qui sont en mi-temps thérapeutique, qui sont proches de la retraite, donc c'est vrai que nous, nos seniors, on les privilégie, on leur facilite le travail, pour l'instant, par rapport aux seniors $q u$ 'on peut embaucher de l'extérieur ». La diversification des recrutements vers les seniors et les handicapés - n'est donc pas d'actualité.

Mais le caractère endogène des recrutements s'inscrit également ici dans ce paternalisme à la française marqué par le clivage national/étranger (Noiriel, 1988). Ce restaurant KFT a été repéré par les intermédiaires de l'emploi pour sa préférence nationale, confirmée par ce salarié de trente d'ancienneté, délégué CFDT du restaurant: "Ici, j'ai remarqué ça, depuis plusieurs années, on ne recrute que des Français ". Or, cette préférence coupable a été mise à rude épreuve avec la MRS, utilisée dans le cadre du partenariat KFT-Pôle Emploi. En effet, la méthode oblige les employeurs à recevoir des candidats venus d'horizons inconnus mais ayant réussi les exercices, et à pourvoir leur offre avec l'un d'entre eux. Elle les contraint à une interaction qui crée, avec plus ou moins de succès, les conditions de recrutements improbables.

Mme $Y$ est ainsi devenue, à son insu, l'héroïne de l'histoire phare de l'agence de services spécialisés de Pôle Emploi enquêtée : en dépit de ses réticences face aux candidats ayant réussi les exercices, elle a recruté d'un employé d'origine malgache. Voici le récit que nous en fait la responsable de l'équipe MRS : "Elle me rappelle et me dit : "oh, je crois qu'il y a un souci, sur la liste, il n'y en a aucun de Français". Je lui dis "oh, je suis étonnée, écoutez, on va reprendre le fax" et puis je le prends, et il n'y a que des noms à consonance étrangère. Alors je dis "écoutez, j'ai la même liste que vous, et ils sont Français, je confirme, et quand bien même ils ne le seraient pas, leur titre de séjour est en règle, ils ont le droit de travailler sur le 
territoire français" - "oui, mais bon, vous voyez bien ce que je veux dire ?" - "Non, je ne comprends pas du tout, je vous assure qu'ils ont tous réussi les exercices, ils ont tous les habiletés", "oui, bon, bon, bon", qu'elle me dit, et là je me dis "houla, c'est pas gagné". Et elle les a tous pris en entretien, et il s'avère qu'elle a pris celui qui avait le nom le plus long!".

A l'instar de ce qu'a souligné Ph. Bataille (1997) pour les stagiaires d'origine maghrébine que leurs formateurs parviennent à placer, en situation d'urgence ou d'imprévu, chez un employeur qui finalement s'en félicite, la MRS crée des opportunités de placement en privant les employeurs de leurs marges de choix habituelles. Reste que cette expérience aura ici été unique: " voilà, là, c'était juste sur ce jeune homme-là, parce que sur la suite, elle nous a fait d'autres coups, elle est vraiment "crrr" "(Responsable MRS). En effet, Mme Y nous a affirmé qu'elle ne souhaitait plus recourir à la MRS, afin de limiter les recrutements en nombre, jugés trop perturbants pour le service, et de se centrer sur les recrutements au fil de l'eau par bouche-à-oreille et sur les candidatures spontanées : " c'est ce qui marche le mieux ». L'endogénéisation des recrutements a donc repris ses droits.

Pour autant, ce restaurant n'est pas " perdu » pour la diversité qui provient, en réalité, des étudiants, cette fenêtre laissant entrer une pluralité de nationalités. Mme $Y$ se félicite de disposer dans son restaurant de quatorze nationalités, dont un "jeune homme noir, [qui] a un master en gestion, étudiant étranger, ça fait huit ans qu'il est chez nous, il va partir au Canada, c'est un gars... il a un contrat étudiant. Il fait 20h par semaine. Il est congolais. Et ce garçon fait des stages depuis six ans, chez tous les huissiers, les notaires, de [la ville], gratuitement. Et pour manger, il vient chez nous. C'est quand même malheureux, non ?". Cette argumentation est intéressante car à double détente. Elle atteste de l'ouverture de l'entreprise qui a su accueillir ce " jeune homme noir » et reconnaître ses " capacités ». Mme $Y$ se défend de l'avoir recruté par charité, tout en suggérant que sans son emploi chez KFT, il serait en grande difficulté. II se pourrait alors qu'il soit aussi apprécié pour sa situation de disqualification sociale, qui confère à Mme $Y$ une grandeur morale et fait de lui un obligé. Reste que ce jeune homme demeure intégré sur le registre provisoire de la catégorie " étudiant ", aux marges du noyau dur stabilisé et bénéficiaire de temps pleins aménagés.

\section{Recrutement exogène, stabilité polyvalente et diversification aux marges}

La constitution de noyaux durs peut prendre d'autres formes. On sait que, dans les chaînes, les exploitants ne sont guère en mesure d'accéder aux réseaux qui se construisent sur la base d'un ancrage local dès lors qu'ils se plient aux mobilités qu'impose leur carrière (MacHatton et al., 1997). Ils encouragent alors les pratiques de cooptation mais se tournent également vers le marché du placement. Mlle $Z$, jeune directrice, mobile et diplômée (Bac +4) d'un restaurant RAP de cinquante personnes, compte ainsi sur ses salariés pour collecter des candidatures. Son dernier recrutement, suite à la démission d'une étudiante ayant terminé ses études, a précisément consisté à recruter l'amie d'une équipière. Mais Mlle $Z$ compte aussi sur les ressources de l'organisation, notamment sur les campagnes de communication organisées par le groupe : "on a tout ce qu'on appelle la PLV [publicité sur le lieu de vente], on a des bâches, des affiches, des mini-CV quand on est en période de recrutement, et le groupe fait pas mal de choses pour se faire connaître (...) Et on utilise internet également ". Le recours au marché passe, en outre, par des coopérations entre directeurs de restaurants d'une même zone géographique, notamment pour les recrutements périodiques qui ont lieu en juin: "quand tous les étudiants ont fini leurs 
études, nous c'est vrai qu'en général on fait des opérations [avec Pôle Emploi], les trois restaurants ensemble ". Comme dans le cas précédent, le marché est systématiquement sollicité pour les recrutements programmables, mais, au-delà des étudiants, les canaux activés visent également les demandeurs d'emploi.

Or, les recrutements par MRS donnent des résultats qui ne sont pas toujours jugés concluants: "beaucoup ne savent pas ce que c'est que de travailler, d'être présent, et d'avoir des règles, et de venir à l'heure, enfin, c'est... voilà. On veut juste le minimum, puisqu'on les forme derrière, c'est ce que je vous dis, ce minimum là je n'arrive pas forcément à le retrouver quand je passe par le recrutement par simulation ». Mais ces critiques n'empêchent pas de continuer à recourir à la méthode, les candidats inconnus ont leur chance même lorsqu'ils ne sont pas étudiants. De fait, les catégorisations ex-ante des postulants sur la base de leurs caractéristiques extra-professionnelles font place ici à des catégorisations ex-post liées à un diagnostic d'employabilité : " nous, on juge absolument pas ni sur la couleur, l'origine ou quoi ", ce que confirment les intermédiaires de l'emploi. Le restaurant étant dernièrement passé halal, il a doublé son chiffre d'affaire avec l'arrivée d'une nouvelle clientèle, sans que cela n'influe sur le recrutement : "Je n'ai absolument pas voulu changer et je n'ai pas voulu tomber dans le stéréotype à mettre que des Musulmans dans mon restaurant, je n'ai rien changé par rapport à ça ».

Car les exigences de Mlle $Z$ sont liées à une politique de polyvalence qu'elle tient à faire partager le plus largement possible. Son problème est de conjurer l'absentéisme d'étudiants " qui n'ont pas forcément la notion du travail ", mais également le turn-over, qui s'élevait à $100 \%$ en 2011. Sa politique consiste, depuis deux ans qu'elle est en poste, à offrir des contrats de travail à temps plein de 30 ou 35 h, à des salariés pour qui ce sera un "métier » et non plus un " job ». Certes, ils sont plus difficiles à remplacer, mais elle y voit un moyen de faire baisser l'absentéisme et de les stabiliser: "ici, j'en ai qui ont plus de dix ans d'ancienneté, par exemple ». L'enjeu n'est pas de faire vieillir les salariés dans l'emploi : " la plus âgée doit avoir 43 ans ", d'autant qu'un temps plein dans la restauration rapide pose rapidement des problèmes de conciliation avec la vie personnelle. Mais il est d'entretenir un noyau dur de " temps pleins " polyvalents, ce qui lui permet, en parallèle, de rechercher des "Handicapés " pour leur offrir des postes aménagés : "J'en ai déjà un qui est plus ou moins polyvalent, il fait les grills, les frites, voilà; il ne veut pas aller en caisse donc on ne le force pas. Et j'en ai un autre qui s'occupe du nettoyage ou de l'entretien de la salle. (...) Et j'essaie d'en trouver d'autres (...) si je peux avoir six ou sept personnes et de tout handicap possible (...) Ça fait aussi partie de ce que je veux dans mon restaurant, de la diversité ".

De fait, les travailleurs handicapés sont le gage d'une certaine stabilité, les deux dont elle dispose "sont là depuis au moins entre 8 et 10 ans ", même s'ils font l'objet d'usages restreints. Ils peuvent alors, aux côté des " étudiants ", exercer dans l'orbite d'un noyau dur de salariés stables polyvalents qui ne sauraient bénéficier, à terme, des avantages de l'âge ou de l'ancienneté - sauf à faire carrière dans l'encadrement. La diversité suppose donc ici l'abolition des privilèges associés à la loyauté. C'est en élargissant la base de salariés flexibles qu'il devient possible d'intégrer des candidats de la diversité normalisée qui le sont moins. Et l'élargissement de cette base flexible passe non seulement par la mobilisation de "cadets sociaux ", femmes et jeunes (Monchatre, 2010), mais également de catégories ethnicisées n'ayant d'autre choix que « l'emploi qui reste » (Eckert, 2011). 


\section{Conclusion}

Le travail est un enjeu de luttes et le recrutement fait partie des opérations qui donnent à voir les modalités de sa distribution sociale. Les recruteurs prospectent, trient, évaluent, contribuant à la sélectivité d'un marché du travail dont ils sont en quelque sorte les gardiens: ils jouent, de fait, un bien mauvais rôle - ainsi qu'en attestent les réactions indignées au documentaire de Didier Cros (2011). Leur enthousiasme à se saisir de la thématique de la diversité peut, à ce titre, être considéré comme le symptôme de leur aspiration à redorer le blason de leur profession. Mais leur action ne saurait être analysée à l'aune de ce seul rôle de vigile, plus ou moins sympathique.

Les recruteurs constituent la partie visible d'un dispositif de mobilisation salariale qui les dépasse amplement mais dont leurs actions en matière de diversité constituent un bon analyseur. Les chaînes étudiées encouragent ainsi le recours à des catégories discriminées, pour des usages différenciés selon la présomption de compétence associée à leur source de discrédit. La promotion de " conventions d'usages restreints" pour des catégories qu'ils ne recrutaient pas jusqu'ici (seniors, handicapés) permet d'invisibiliser les "conventions d'usages flexibles" en vigueur dans leurs emplois de service ouverts aux catégories souffrant de discriminations raciales. Ils s'engagent ainsi dans une normalisation de la gestion des ressources humaines au double sens de mise aux normes modernisatrice et de retour à la normale. De fait, ils pratiquent une diversité « à la carte » (Junter et Senac, 2011), dans la continuité des formes de paternalisme qui caractérisent le secteur. Aînés, cadets et adoptés sont désormais appelés à cohabiter avec les accidentés mis en circulation sur le marché du travail.

Ces politiques de "diversité normalisatrice " parviennent ainsi à entretenir l'invisibilité d'une gestion du travail discriminatoire. Elles s'insèrent dans les logiques productives selon des modalités très éloignées de la promesse d'une performance tirée d'une plus grande mixité des équipes. Loin d'égaliser la condition salariale par une «indifférence aux différences", elles renforcent la différenciation des usages des salariés selon leurs caractéristiques extra-professionnelles, le poids de ces dernières n'étant pas allégé mais, au contraire, banalisé. Des "emplois-jeunes" aux "emplois seniors » en passant par les emplois handicapés, des jobs d'étudiants aux temps partiels féminisés, la diversité ne fait qu'allonger la liste des catégories cibles de l'action publique et privée en faveur de l'emploi flexible, tout en renflouant une armée de réserve dont l'abondance favorise la mobilisation. A ce titre, elle s'ajoute à l'ensemble des dispositifs qui contribuent à l'amère victoire de l'emploi sur la lutte contre les inégalités (Ben Michaels, 2009).

Notre analyse montre enfin que les politiques de diversité modifient moins les pratiques de recrutement en vigueur qu'elles ne se superposent à elles. Elles procèdent en qualifiant des candidats discrédités pour les segments d'activité les moins prisés. Tout en leur procurant des marchepieds vers l'emploi, elles livrent ces réservistes aux mécanismes d'appel et de rejet du marché du travail. Le risque de leur enfermement dans une qualification subalterne, susceptible d'être considérée comme une seconde nature, féminisée, ethnicisée ou handicapée, n'en est que plus grand. Dès lors, si la diversité contribue à raffermir les " différences » sur un registre identitaire (Senac, 2012), c'est moins par leur valorisation individuelle que par leur utilisation collective et tenace dans des emplois dépréciés. Loin 
d'être abolie, la violence des discriminations risque alors de se perpétuer sous la forme d'un enclavement tout au long de la vie.

\section{Bibliographie}

Aberg R., 2001. - "Equilibrium unemployment search behavior and unemployment persistency ", Cambridge Journal of Economics, 25, 131-147.

Addison J., Portugal P., 2002. - " Job research methods and research outcomes", Oxford Economic Papers, 54, 3, pp. 505-533.

Alaluf M., 2000. - "Le travail du temps ", in G. de Terssac et G. Tremblay (Eds), Où va le temps de travail ? Toulouse, Octarès, pp. 203-213.

Amadieu J.-F., 2008. - "Vraies et fausses solutions aux discriminations ", Formation Emploi, 101, pp. 89-104.

Bataille P., 1997. - Le racisme au travail, Paris, La Découverte.

Balazs G., Faguer J.-P., 1979. - " Jeunes à tout faire et petit patronat en déclin ", Actes de la recherche en sciences sociales, 26-27, pp. 49-55.

Benn Michaels W., 2009. - La diversité contre l'égalité, Raisons d'agir, traduction française.

Bereni L., 2009. - " "Faire de la diversité une richesse pour l'entreprise" : la transformation d'une contrainte juridique en catégorie managériale ", Raisons politiques, 35, 3, pp. 87-105.

Bereni L., Jaunait A., 2009. - " Usages de la diversité », Raisons politiques, 35, 3, pp. 5-9.

Bessy C., Marchal E. 2007. - L'usage des canaux de recrutement par les entreprises, Document de travail $n^{\circ} 89$, CEE.

Bouchardeau C., Capelier T., 2010. - Evaluation de la méthode de recrutement par simulation. Usage par les entreprises et impact sur les pratiques de recrutement, Repères et analyses $n^{\circ} 16$, Pôle Emploi, Juillet.

Bourdieu P., 1980. - « Le capital social. Notes provisoires ». Actes de la recherche en sciences sociales, 3, pp. 2-3.

Bureau M.-C., Marchal E., 2009. - «Incertitudes et médiations au cœur du marché du travail », Revue Française de Sociologie, 50, 3, pp. 573-598

Caradec V., Lefrançois C., Poli A., 2009. - "Quand la discrimination et la diversité se déclinent selon l'âge : émergence, appropriation et ambivalences ", Cahiers internationaux de sociologie, 127, 2, pp. 223-245.

Cartier M., 2001. - " Nouvelles exigences dans les emplois d'exécution des services publics. L'observation des épreuves orales du concours de facteur ", Genèses, 42, pp. 72-91.

Cros D., 2011. - La gueule de l'emploi, Film Zadig Productions.

Daniellou F., 2002. - "Le travail des prescriptions ", Actes du $17^{\text {ème }}$ congrès de la SELF (Société d'ergonomie de langue française), Aix en Provence, conférence inaugurale, pp. 8-15. Datchary C., 2011 - La dispersion au travail, Toulouse, Octarès.

Devetter F.-X., 2006. - « La disponibilité temporelle au travail des femmes : une disponibilité sans contrepartie ? ", Temporalités [En ligne], 4 | 2006, mis en ligne le 29 juin 2009, consulté le 07 septembre 2012. URL : http://temporalites.revues.org/366

Dobbin F., 2009 - Inventing Equal Opportunity, Princeton, Princeton University Press.

Doytcheva M., 2009. - "Réinterprétations et usages sélectifs de la diversité dans les politiques des entreprises ", Raisons politiques, 35, 3, pp. 107-123.

Doytcheva M., 2010. - « Usages français de la notion de diversité : permanence et actualité d'un débat ». Sociologie, 1, 4, pp. 423-438 
Dubernet A.-C., 1996. - « La sélection des qualités à l'embauche. Une mise en scène de la valeur sociale ", Formation Emploi, 54, pp. 3-14.

Eckert H., 2011. - " Discrimination et reproduction sociale ou l'emploi qui reste... ", Agora Débats/Jeunesse, 57, pp. 107-120.

Eymard-Duvernay F. (Dir.), 2012. - Epreuves d'évaluation et chômage, Toulouse, Octarès.

Eymard-Duvernay F., Marchal E., 1997. - Façons de recruter. Le jugement des compétences sur le marché du travail, Paris, Métailié.

Forté M., Monchatre S., 2013. - Recruter dans l'hôtellerie-restauration : quelle sélectivité sur un marché du travail en tension ? Revue de I'IRES, n76, p.127-150.

Gaudemar de J.-P., 1979. - La mobilisation générale, Paris, Editions du champ urbain.

Hanique F., 2004. - Le sens du travail. Chronique de la modernisation au guichet. Toulouse, Eres.

Godbout J., 1995 - L'esprit du don. Montréal, Boréal.

Goffman E., 1977. - Stigmates. Les usages sociaux des handicaps. Paris, Minuit, traduction française.

Granovetter M.-S., 1973. - « The Strength of weak ties », American Journal of Sociology, 78, 6, pp. 1360-1380.

Guilbert M., 1966. - Les fonctions des femmes dans l'industrie, Paris, La Haye Mouton.

Guiraudon V., 2004. - "Construire une politique européenne de lutte contre les discriminations : I'histoire de la directive "RACE" ॥, Sociétés contemporaines, 53, pp. 11-32.

Hidri 0., 2008. - «Faut-il travailler son corps pour réussir un entretien d'embauche ? La place de l'apparence physique dans les manuels d'Aide à la recherche d'emploi ", Lien social et Politiques, 59, pp. 21-32.

Hidri 0., 2009. - "Qui se ressemble s'assemble... " : Le recrutement des jeunes diplômés dans les professions de cadre commercial ॥, Formation emploi, 105, pp. 67-82.

Hochschild A., 1983. - The Managed Heart. Commercialization of Human Feelings. Berkeley, University of California Press.

Junter A., Sénac-Slawinski R., 2011. - "La diversité : sans droit ni obligation ", in Les discriminations entre les femmes et les hommes, Paris, Presses de Sciences Po, pp. 199-231.

Kergoat K., 1982. - Les ouvrières, Paris, Le Sycomore.

Lagarenne C. et Marchal E., 1995. - Recrutements et recherche d'emploi, La lettre du CEE, $\mathrm{n}^{\circ} 38$.

Lanquetin M.-T., 2009. - "Egalité, diversité et discriminations multiples ", Travail, genre et société, 21 (1), pp. 91-106.

Larquier G. (de), Marchal E., 2008. - Le jugement des candidats par les entreprises lors des recrutements, Document de travail $n^{\circ} 109$, CEE.

Lavitry L., 2012. - " Le jugement d'employabilité : un nouveau savoir pour gérer les chômeurs ? ", Sociologies pratiques, 24 (1), p. 53-65.

Laurens S., Neyrat F. (Dir.), 2010. - Enquêter, de quel droit? Menaces sur l'enquête en sciences sociales, Paris, Le Croquant.

Linhart R., 1978. - L'établi. Paris, Minuit.

Lockyer C., Scholarios D., 2004. - « Selecting hotel staff : why best practice does not always work ", International Journal of Contemporary Hospitality Management, Vol. 16, n², pp. 125-135.

MacHatton M.T., VanDyke T., Steiner R., 1997. - « Selection and retention of managers in the US restaurant sector ", International Journal of Contemporary Hospitality Management, Vol. 9, n4, pp. 155-160. 
Marchal E., Rieucau G., 2009. - « Formes d'intermédiation et formes de sélection : les contrastes entre annonces et réseaux de relations ", Economie et Sociétés, Série Socioéconomie du travail, $A B, 30$, pp. 3-26.

Marchal E., Rieucau G., 2010. - Le recrutement, Paris, La Découverte, Collection Repères. Marx K., [1867] 1969. - Le capital, Paris, Gallimard, Folio.

Maruani M. et Nicole C., 1989. - Au labeur des dames. Métiers masculins, emplois féminins, Paris, Syros Alternatives.

Maruani M., Reynaud E., [1993] 2004. - Sociologie de l'emploi, La Découverte, Repères.

Monchatre S., 2007. - "Des carrières aux parcours... en passant par la compétence ", Sociologie du travail, 49, 4, pp. 514-530.

Monchatre S., 2010. - Etes-vous qualifié pour servir ? Paris, La Dispute.

Naville, P., 1972. - Temps et technique. Les structures de la vie de travail. Genève, Droz.

Noiriel G., 1988. - Le creuset français, Paris, Seuil.

Nkouitchou Nkouatchet R., 2005. - «La précarité de l'emploi au service de la prospérité du fast-food ", Sociologie du travail, 47, 4, pp. 470-484.

Papinot C., 2011. - « La motivation, nouvelle inflexion rhétorique dans la mobilisation de la main-d'œuvre? L'exemple du marché du travail intérimaire en France ", Revue internationale de sociologie, 21, 2, pp. 301-314.

Petersen T., Saporta I., Seidel M.D., 2000. - "Offering a job: meritocracy and social networks ", American Journal of Sociology, 106, 3, p. 763-816.

Pitti L., 2005. - « Catégorisations ethniques au travail. Un instrument de gestion différenciée de la main-d'œuvre ", Histoire \& mesure, XX, 3/4, pp.69-101.

Point S., 2010. - « Communiquer sur la diversité. L'exemple des grands groupes hôteliers » Revue Française de gestion, 206, 7, pp. 49-64.

Puech I., 2006. - « Femmes et immigrées, corvéables à merci », Travail, Genre et Société, $\mathrm{n}^{\circ} 16$, p. 39-51.

Richebé N., 2002. - « Les réactions des salariés à la logique compétence : vers un renouveau de l'échange salarial ? » Revue Française de sociologie, 43, 1, p. 99-126.

Rieucau G., 2012. - "À propos de « La Gueule de l'emploi », La nouvelle revue du travail [En ligne], 1 | 2012, mis en ligne le 10 décembre 2012, consulté le 16 janvier 2013. URL : http://nrt.revues.org/411

Robert-Demontrond P., Joyeau A., 2010. - "La performance des politiques de diversité en question : une étude de la représentation des acteurs ", Management et Avenir, 31, pp. 7191.

Senac R., 2012. - L'invention de la diversité, Paris, PUF

Theurier J.-P., 1983. - Etude des modes de sélection à l'embauche dans le secteur privé, Thèse de Doctorat, LAST-CNRS, juin.

Warhurst C., Nickson D., 2007. - " Employee experience of aesthetic labour in retail and hospitality ", Work, Employment \& Society, Vol 21 (1), p. 103-120.

Zajdela H., 2009. - « Comment et pourquoi activer les inactifs ? » Travail et emploi, 118, pp. 69-76.

\section{Documents cités :}

- Charte de la diversité en entreprise, 2011, Bilan Diversité. Pratiques des entreprises signataires de la charte de la diversité - 2011, Cabinet Inergie, en ligne à cette adresse : http://www.charte-diversite.com/docs/data/outils/documents/Bilan-Diversite-2011.pdf 
- IMS-Entreprendre pour la Cité, 2004, Non-discrimination et gestion de la diversité dans les entreprises en France, Paris, publication de I'IMS.

- IMS-Entreprendre pour la Cité, 2005, «Ressources Humaines, Non-Discrimination et Diversité : Recrutement ", Groupe de travail inter-entreprises, Cycle 2004-2005, EqualLatitude.

- IMS-Entreprendre pour la Cité, 2008, Vers l'entreprise inclusive ? Les six clés de la diversité, Paris, Editions Autrement.

- IMS-Entreprendre pour la Cité, 2010, «Diversité du capital humain et performance économique », Rapport réalisé par Goodwillmanagement. 